\title{
Critical role for Orai1 C-terminal domain and TM4 in CRAC channel gating
}

\author{
Raz Palty ${ }^{1}$, Cherise Stanley ${ }^{1}$, Ehud Y Isacoff ${ }^{1,2,3}$ \\ ${ }^{I}$ Department of Molecular and Cell Biology, University of California Berkeley, Berkeley, CA 94720, USA; ${ }^{2}$ Helen Wills Neurosci- \\ ence Institute, University of California Berkeley, Berkeley, CA 94720, USA; ${ }^{3}$ Physical Bioscience Division, Lawrence Berkeley \\ National Laboratory, Berkeley, CA 94720, USA
}

Calcium flux through store-operated calcium entry is a major regulator of intracellular calcium homeostasis and various calcium signaling pathways. Two key components of the store-operated calcium release-activated calcium channel are the $\mathrm{Ca}^{2+}$-sensing protein stromal interaction molecule 1 (STIM1) and the channel pore-forming protein Orai1. Following calcium depletion from the endoplasmic reticulum, STIM1 undergoes conformational changes that unmask an Orail-activating domain called CAD. CAD binds to two sites in Orai1, one in the $\mathrm{N}$ terminal and one in the $\mathrm{C}$ terminal. Most previous studies suggested that gating is initiated by STIM1 binding at the Orai1 $\mathrm{N}$-terminal site, just proximal to the TM1 pore-lining segment, and that binding at the C terminal simply anchors STIM1 within reach of the $\mathbf{N}$ terminal. However, a recent study had challenged this view and suggested that the Orail $\mathrm{C}$-terminal region is more than a simple STIM1-anchoring site. In this study, we establish that the Orai1 C-terminal domain plays a direct role in gating. We identify a linker region between TM4 and the C-terminal STIM1-binding segment of Orai1 as a key determinant that couples STIM1 binding to gating. We further find that Proline 245 in TM4 of Orai1 is essential for stabilizing the closed state of the channel. Taken together with previous studies, our results suggest a dual-trigger mechanism of Orai1 activation in which binding of STIM1 at the N- and C-terminal domains of Orai1 induces rearrangements in proximal membrane segments to open the channel.

Keywords: CRAC channel; Orai1; stim1; gating; calcium

Cell Research (2015) 25:963-980. doi:10.1038/cr.2015.80; published online 3 July 2015

\section{Introduction}

The store-operated calcium release-activated calcium (CRAC) channel has two essential components, stromal interaction molecule 1 (STIM1), the endoplasmic reticulum (ER) $\mathrm{Ca}^{2+}$ sensor and Orail (also known as CRACM1), the plasma membrane (PM) channel pore-forming subunit [1-10]. STIM1 is a single-pass ER membrane protein with several functional domains including an EF hand calcium-binding domain and a sterile $\alpha$ motif (SAM) [11-13] that face the ER lumen and a CRAC channel activation domain called CAD [14] or

Correspondence: Ehud Y Isacoff

Tel: +510-642-9853;

E-mail: ehud@berkeley.edu

Received 3 February 2015; revised 30 March 2015; accepted 22 May

2015; published online 3 July 2015
SOAR [15] (spanning residues 339-448 in hSTIM1, referred to hereafter only as CAD) facing the cytosol. Each Orail protein has four transmembrane segments and a hexameric assembly $[16,17]$ of Orai1 subunits forms cation-conducting channels in the PM $[6,9,18]$. Much is known about the coupling between ER calcium depletion and CRAC channel activation $[7,19,20]$. The process begins when depletion of calcium from the ER leads to calcium disassociation from the EF hand of STIM1, facilitating dimerization of SAM domains [21]. Dimerization of STIM1 ER luminal domains triggers extensive conformational changes in the cytoplasmic domains that lead to the exposure of CAD [20, 22-26]. CAD binds with low affinity to a site in the Orail $\mathrm{N}$ terminal (NBD, residues 73-87; Figure 1A) and with higher affinity to a site in the Orai1 C terminal (CBD, residues 267-292; Figure 1A) $[14,15,28,29]$. The interaction of activated STIM1 with Orai1 results in clustering of STIM1-Orai1 at sites of ER- 
PM interface and in pore opening of CRAC channels that mediates localized and selective calcium influx into cells $[3,9,30-35]$. A defining event in this sequence is the coupling of STIM1 binding with opening of the CRAC channel pore. However, this process is not well understood. Structure-function studies have indicated that the first transmembrane segment (TM1) of Orail lines the channel pore [36, 37]. The crystal structure of Drosophila melanogaster Orai (dOrai), an ortholog of mammalian Orai1, revealed that the NBD and CBD project into the cytoplasm, with the NBD forming a continuous helical extension of TM1 that is located near the center of the channel and with the CBD forming a helical extension of TM4 that is connected to TM4 via a conserved linker region, which is located at the periphery of the channel (Figure 1A and see [38]). The structure further showed that CBDs from neighboring Orail subunits pair with one another through hydrophobic coiled-coil interactions that involve residues Ile316 and Leu319 (L273 and L276 in hOrai1) from each subunit. Structure-function studies identified mutations in the Orail NBD [37, 39] and CBD [40-42] that abolish channel activation, yet, of these, only CBD mutants abolished the physical interaction with STIM1. These findings led to the formulation of the Anchoring Model of Orai gating (Figure 1B and see [43]), in which CAD binds to a high-affinity site in the CBD to anchor STIM1 to the channel, and activation is induced when CAD binds to a low-affinity site in the NBD. Supporting the notion that the role of the Orail CBD is to anchor STIM1 a recent study [43] generated an Orail CBD truncation mutant that could not be activated by STIM1, presumably because the mutant lacked a substantial piece of the CBD coiled-coil region and thus could not bind STIM1; and this Orai1 truncation was chronically activated when the CAD was directly linked to the shortened CBD. In contrast, another study [44] found that a nearby, but distinct CBD deletion mutant could not be activate even if CAD was directly linked to the mutated CBD, suggesting that the Orail CBD may have a function beyond just anchoring of STIM1 and that the two Orai1 termini may operate as an ensemble to drive gating (Figure $1 \mathrm{C}$ and see [44]).

To investigate the mechanism of STIM1-induced gating of Orai1, we relied on an earlier approach [45], which we extended here to distinguish the effect of mutations on affinity for STIM1 versus gating. The approach was to anchor a STIM1 fragment similar to CAD to one normal terminal while mutating the other terminal, thereby ensuring that the local concentration of the STIM1 fragment and its ability to interact with the normal terminal is kept constant (Figure 2A). Our experiments identify regions within the Orail NBD and CBD whose deletion
A

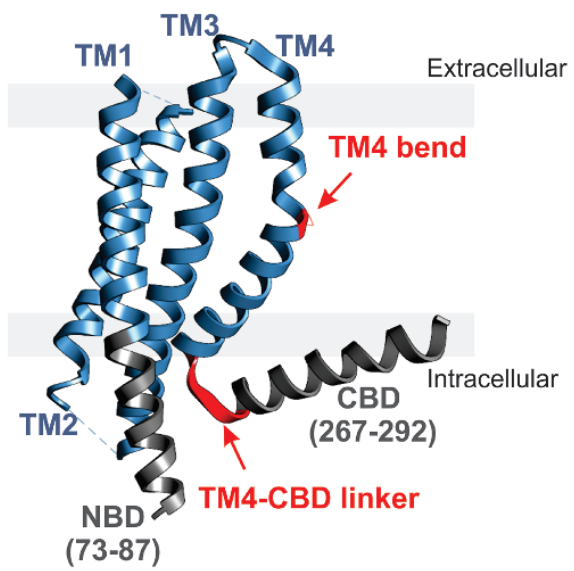

B
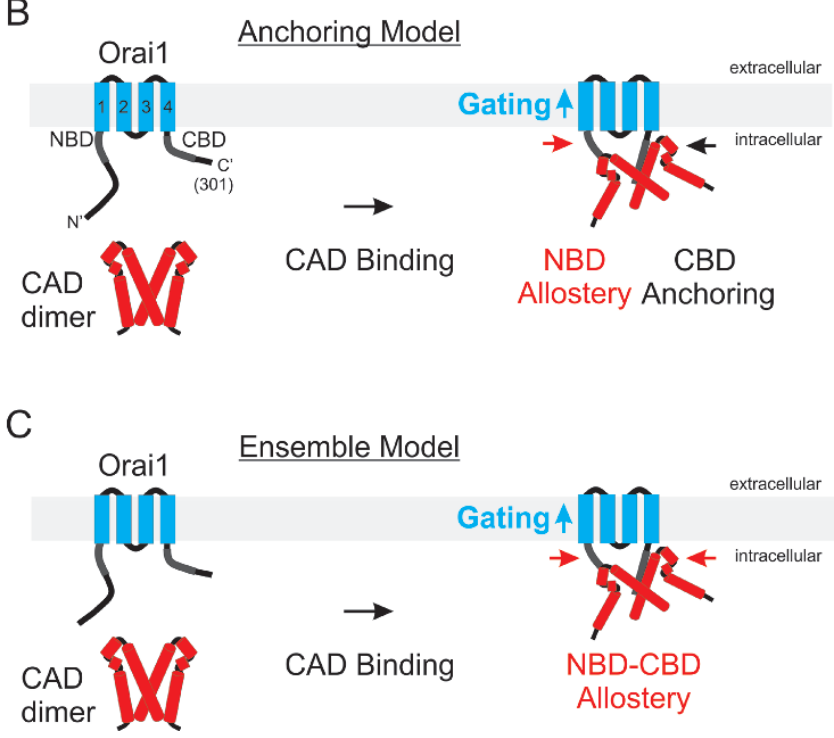

Figure 1 Models of STIM1-induced activation of Orai1 channels. (A) Cartoon representation of a monomer from the crystal structure of Drosophila Orai (PDB ID-4HKR). The transmembrane segments TM1-4 are colored in cyan, the NBD and CBD in grey. (B) The Anchoring Model. CAD binding to a high-affinity site in the CBD serves to anchor STIM1 to the channel but has no direct effect on channel gating. STIM1-Orai1 interaction that directly affects channel gating occurs when CAD binds to a low-affinity site in the NBD, which opens the channel pore. (C) The Ensemble model. Orai1 channels are activated by simultaneous binding of CAD to both of Orai1 NBD and CBD. Simultaneous binding of STIM1 to both of Orai1 NBD and CBD supplies the energy for channel gating by pulling on membrane proximal regions at both termini of Orai 1 . 
eliminates activation by free STIM1 fragment, but where linkage of the STIM1 fragment restores activation, indicating a role in STIM1 binding. We also identify regions in both the NBD and CBD whose elimination cannot be rescued by linkage of the STIM1 fragment, suggesting a fundamental role not only for the N-terminal domain but also for the $\mathrm{C}$-terminal domain in channel gating. Further investigation identifies three regions at the junction between TM4 and the CBD that are critical for gating. We find that the hydrophobicity of CBD residues L273 and L276 has a critical role in both STIM1 binding and channel gating. Closer to the membrane, a linker region between the CBD and TM4 contains residues whose mutation abolishes gating without eliminating STIM1 binding. Last, substitution of a conserved proline residue (P245), located at a bend in the middle of TM4 (21), with all other amino acids opens the channel in the absence of STIM1, suggesting that P245 operates as a switch for channel activation. Our results lead to a hypothetical model in which a key step to channel opening is that STIM1 binding to the Orai1 NBD and CBD induces a conformational change that propagates "up" through the TM4-CBD linker into TM4 and modulates TM4 at the P245 switch to drive channel opening.

\section{Results}

Minimal Orail CBD region required for physical and functional interaction with soluble SIC

To study the role of the Orail CBD in channel gating, we initially set out to define the minimal region in the Orail CBD that is required for channel activation by STIM1. Residues 272-292 of Orai1 were recently shown to encompass a degradation resistant polypeptide that binds to part of the activating domain of STIM1 [46]. Previous studies had also shown that STIM1-dependent channel activity is preserved in a mutant Orail truncated to residue 283 but lost upon truncation to residue 277 or following deletion of residues 272-279 [43, 44, 47]. We therefore made $\mathrm{C}$-terminal truncation mutants of Orai1 ending at D284, A280, L276, E275 and E272 and tested the ability of these truncated versions of Orail to physically and functionally interact with a short STIM1 fragment similar to CAD (S1C, residues 343-465). To study the physical interaction between the Orail CBD truncation mutants and S1C in cells, we employed co-localization and Förster resonance energy transfer (FRET) analyses. Co-localization of S1C with Orail was determined in HEK293 cells co-expressing EYFP-tagged S1C (EYFP-S1C) together with mCherry-tagged full-length or CBD-truncated versions of Orail (mCherry-Orail or mCherry-Orai $\Delta \mathrm{C}$ ) by comparing PM and intracellular fluorescent intensity profiles. FRET was determined by analyzing the recovery of donor fluorescence after acceptor photobleaching between (donor) EGFP-tagged S1C and (acceptor) mCherry-Orail or mCherry-Orai $1 \Delta \mathrm{C}$. In agreement with previous studies, we found that progressive deletion of the $C$ terminus to residue $276\left(\Delta \mathrm{C}_{277-301}\right)$ reduced both co-localization as well as FRET (Figure 2B, 2C and Supplementary information, Figure S1). Unexpectedly, interaction with S1C was not eliminated in Orai1 $\Delta \mathrm{C}_{277-301}$, whereas additional removal of leucine $276\left(\Delta \mathrm{C}_{276-301}\right)$ abolished the interaction (Figure $2 \mathrm{~B}, 2 \mathrm{C}$ and Supplementary information, Figure S1). The results are consistent with recent structural findings of the STIM-Orai association pocket [46] and suggest that the

Figure 2 Directly linking S1C to Orai1 rescues channel activity in some NBD or CBD truncation mutants but fails in others. (A) Cartoon illustration of the Orai1 constructs used in this work. NBD and CBD regions are shaded in grey, pore residue V102 marked in yellow and tethered STIM1 fragments are marked as red rectangles. (B) Fluorescent images of a cell expressing EGFP-S1C (donor) and mCherry-Orai1 (acceptor) before and after acceptor photobleaching within the indicated region (white box). (C) Bar diagram shows summary of FRET efficiency values measured in cells expressing EGFP-S1C together with the indicated mCherry-Orai1 construct (Purple, $n=13-24$ cells, $\left.{ }^{* *} P<0.01\right)$ and of current densities (Grey, $n=5-15$ cells, ${ }^{*} P<0.05$ ) recorded from cells expressing S1C together with the indicated mCherry-Orai1 constructs. (D) representative plots of the current-voltage relationship of currents recorded from cells in C. (E) Left, summary of current densities recorded from cells expressing the indicated Orai1-SS-EGFP construct or from cells expressing mCherry-S1C together with EGFP-Orai1 $\Delta \mathrm{N}_{1-76}(n$ $=4-7$ cells, $\left.{ }^{* *} P<0.01\right)$. Right, representative plots of the current-voltage relationship of currents recorded from cells expressing Orai1-SS-EGFP $\Delta \mathrm{N}_{1-76}$ and Orai1-SS-EGFP $\Delta \mathrm{N}_{1-77}$. $(\mathrm{F})$ Left, summary of current densities recorded from cells expressing mCherry-SS-Orai1 with the indicated C-terminal deletion (mean \pm SEM of $5-10$ cells, ${ }^{*} P<0.05$ ). Right, representative plots of the current-voltage relationship of currents recorded from cells expressing mCherry-SS-Orai1 $\Delta \mathrm{C}_{276-301}$ and mCherry-SSOrai1 $\Delta \mathrm{C}_{273-301}$. (G) Summary of current densities recorded from cells expressing the indicated Orai1-SS-EGFP or mCherrySS-Orai1 constructs with or without the V102A pore mutation ( $n=5-12$ cells). (H) Representative fluorescent images of NFAT-EGFP in cells co-expressing NFAT-EGFP together with the indicated mCherry-SS-Orai1 construct. Arrows indicate cells with nuclear localization and asterisks indicate cytosolic localization of NFAT. (I, J) Basal Ca ${ }^{2+}$ levels $(n=23-76$ cells) and quantification of nuclear localization of NFAT ( $n=91-268$ cells) in HEK293 cells transfected with the indicated mCherrySS-Orai1 (I) or Orai1-SS-EGFP (J) constructs. 
A
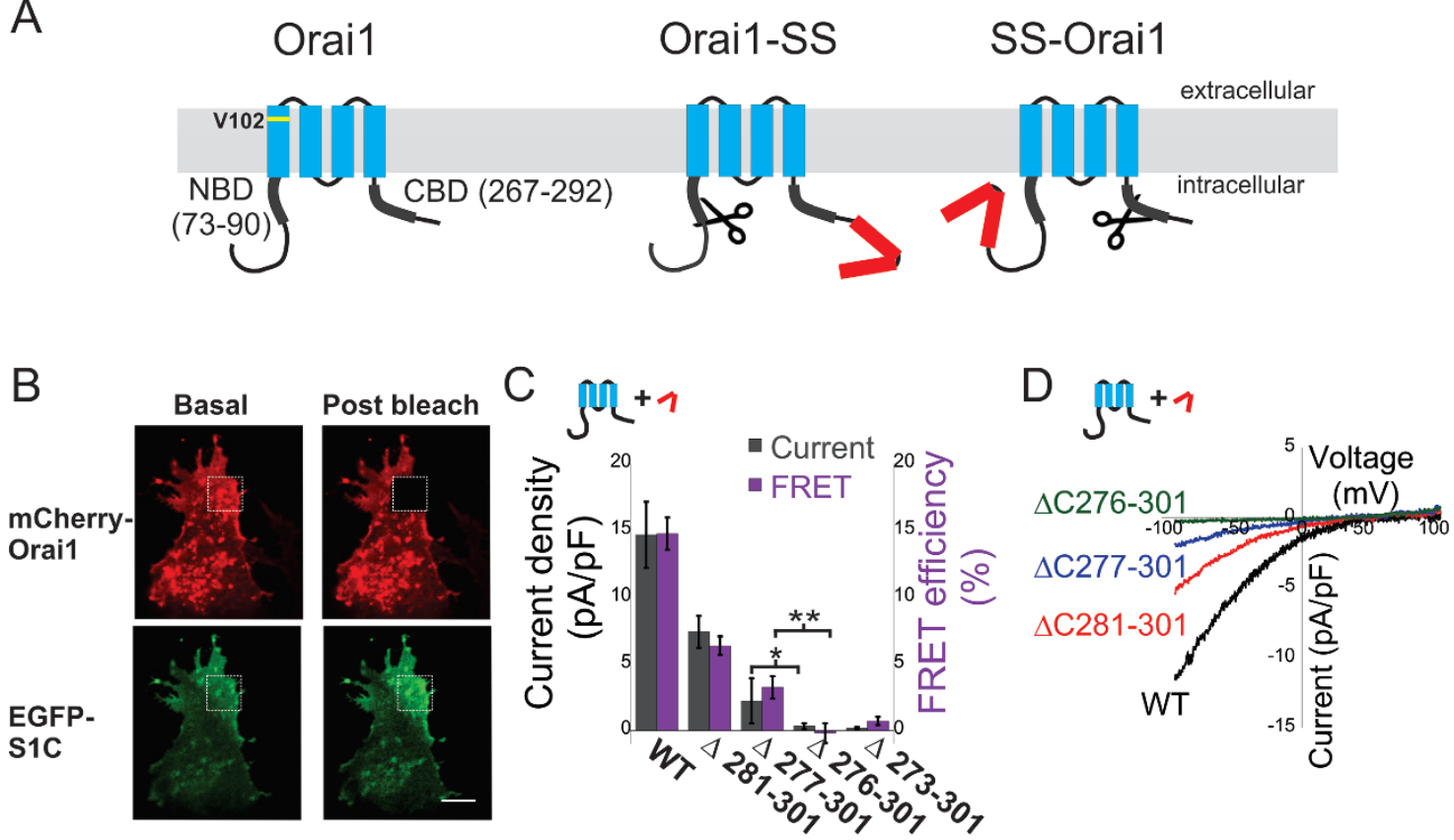

E

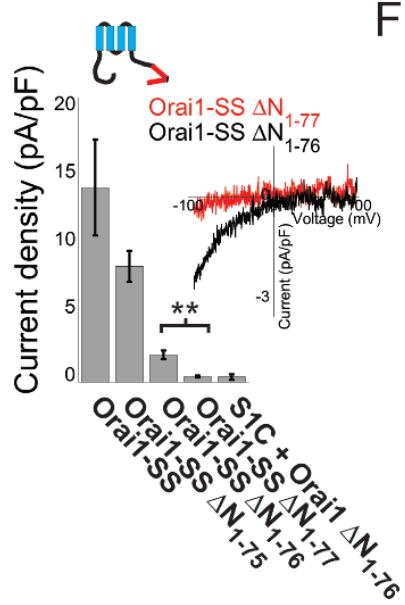

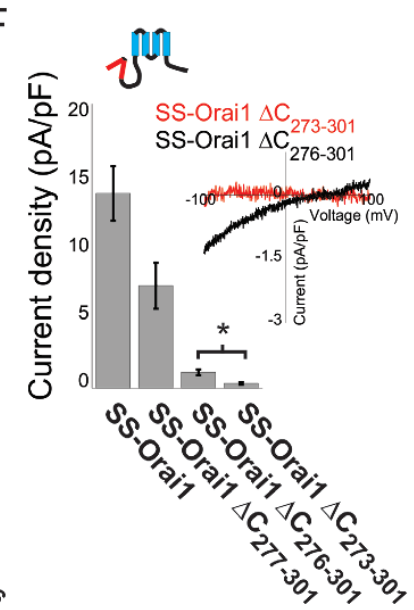

$G$ or or on

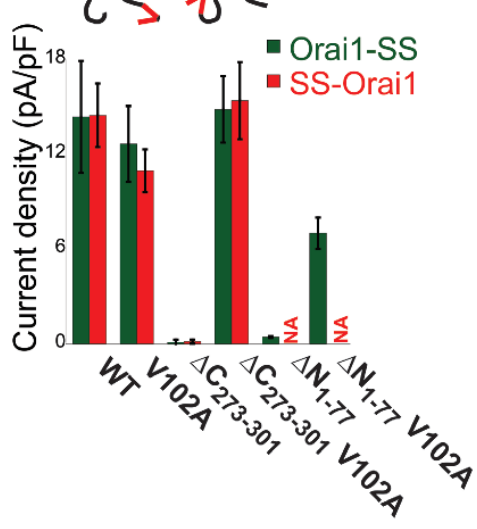

$\mathrm{H}$

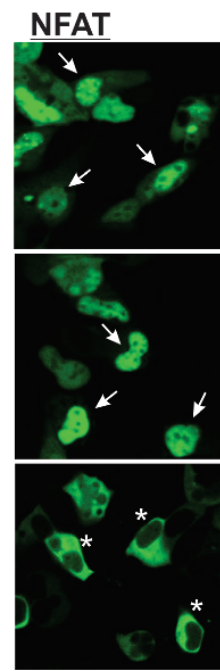

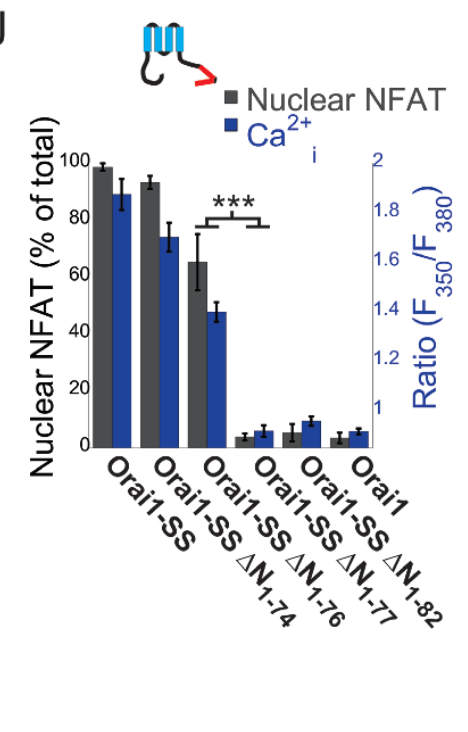


CBD region extending on the membrane proximal side to leucine 276 plays a special role in S1C-Orail interaction. We next examined the effect of the Orail CBD truncation mutants on the activation of channels by S1C. We determined channel activity in two ways: (i) whole-cell patch-clamp measurement of membrane current from individual cells, and (ii) optical measurement of intracellular $\mathrm{Ca}^{2+}$ levels from groups of cells. These functional measurements revealed a similar pattern of behavior to the co-localization and FRET experiments, with progressive deletion of the $C$ terminus up to residue $276\left(\Delta \mathrm{C}_{277-301}\right)$ diminishing channel activity and the additional removal of leucine $276\left(\Delta \mathrm{C}_{276-301}\right)$ completely abolishing it (Figure 2C, 2D and Supplementary information, Figure S1). We further found that while the Orail CBD deletion mutant that is missing amino acids 277-301 retained functional interaction with soluble S1C, its activation by full-length STIM1 was strongly diminished (Supplementary information, Figure S2). To ensure that the reduced channel activity that we observed reflected a reduction in channel activation, rather than an effect on channel expression, we examined the effect of the Orail mutations on expression. All of the CBD deletion constructs exhibited similar expression levels and PM localization as full-length Orai1 (Supplementary information, Figure S1). Moreover, introduction into each of the truncation constructs of the pore mutation V102A, which constitutively opens the channel in the absence of STIM1, restored conductance to both the $\Delta \mathrm{C}_{276-301}$ and $\Delta \mathrm{C}_{273-301}$ Orail mutants, indicating proper channel folding, assembly and capacity to function even though activation by soluble S1C was completely lost (Supplementary information, Figure S1). Together with additional findings, which are described below, these results suggest that while Orail CBD residues 272-284 stabilize the interaction with $\mathrm{S} 1 \mathrm{C}$, a region stretching from the Orai1 TM4 to leucine 276 is essential for physical interaction with, and activation by, soluble S1C.

\section{Direct role in CRAC channel activation for interaction of SIC with both NBD and CBD}

A recent study found that $\mathrm{N}$-terminal truncations of Orail to residue S75 retained STIM1-dependent channel activity, but that further deletion past this point abolished activity [28]. In light of the differences in efficacy in Orail activation between the full-length STIM1 and $\mathrm{CAD}$, we first confirmed this key observation and found that the truncation mutant (Orail $\Delta \mathrm{N} 1-76$ ) displays normal PM expression but could not be activated by S1C (Figures 2E and Supplementary information, Figure S3). Our truncation analysis of the Orail CBD showed that loss of activation by S1C was associated with loss of interaction with S1C. To distinguish between effects of
Orai1 mutations on S1C binding and the ability of S1C, once bound, to activate the channel, we fused S1C (which we label as "S") directly to Orail. We linked a tandem pair of S1C (as the operational unit of STIM1 is a dimer) to Orail either at the $\mathrm{N}$ terminus (SS-Orai1) or $\mathrm{C}$ terminus (Orai1-SS) (Figure 2A). Because deletion of the $\mathrm{N}$ or $\mathrm{C}$ terminus of Orail may introduce steric hindrance that would prevent an attached S1C from reaching its binding domain, we initially confined ourselves to fusions of the tandem S1C fragments to the full-length $\mathrm{N}$ terminus of Orail (SS-Orai1) when we examined CBD deletion mutations or to the full-length $\mathrm{C}$ terminus (Orail-SS) when we examined NBD mutations. When expressed in HEK293 cells, SS-Orail and Orail-SS localized to the PM and were constitutively active, with similar current densities, although some of SS-Orail was retained intracellularly (Figure 2E-2G and Supplementary information, Figure S2). As shown earlier for Orai1-SS [45], and confirmed here, SS-Orail mediated currents that exhibited characteristics of native CRAC current, including an inwardly rectifying current-voltage relationship, high $\mathrm{Ca}^{2+}$ selectivity, permeability to $\mathrm{Na}^{+}$in divalent-free solution and blockade by $\mathrm{La}^{3+}$ (Supplementary information, Figure S2). Moreover, current mediated by SS-Orail was not further increased by co-expression of soluble S1C indicating that the tethered S1C modules induced maximal channel activation. Remarkably, three functional measures of Orail activity - membrane current recording, fura-2 measurement of intracellular calcium and nuclear translocation of the nuclear factor of activated T cells (NFAT) - all revealed some restoration of activity to the Orail $\Delta \mathrm{N}_{1-76}$ and Orail $\Delta \mathrm{C}_{276-301}$ truncation-disabled mutants by molecular linkage of the S1C pair to the other full-length terminal (Figure 2E-2J and Supplementary information, Figure S3). However, we wanted to rule out the possibility that the restored activity arises from the activation of endogenous channels by the S1Cs that are brought to the PM by virtue of their attachment to the mutant Orail channel. To address this, we introduced pore mutations that block Orail currents into the Orail construct to which the $\mathrm{S} 1 \mathrm{C}$ was linked. R91W and E106Q mutations in SS-Orai1 $\Delta \mathrm{C}_{276-301}$ abolished channel activity (Figure 2I), indicating that the activity detected in cells expressing SS-Orail $\Delta \mathrm{C}_{276-301}$ was, indeed, due to $\mathrm{Ca}^{2+}$ conduction through this $\mathrm{S} 1 \mathrm{C}$-fused truncated version of Orail.

Next, we made another set of truncation mutants by removing residues 1-77 from Orail-SS or 273-301 from SS-Orail. These $\mathrm{N}$ or $\mathrm{C}$ truncations abolished channel activity, even when the pair of S1Cs was fused to the remaining full-length terminal of Orail (Figure 2E-2J and Supplementary information, Figure S3). To verify that 
loss of activity in the case of CBD deletion $\Delta \mathrm{C}_{273-301}$ did not depend on the location of the fused S1Cs, we also analyzed the case of C-terminally attached S1Cs and found that in this context CBD truncation of residues 273-301 (Orail-SS $\Delta \mathrm{C}_{273-301}$ ) also completely abolished channel activity (Figure 2G). The loss of activity did not result from incorrect protein folding or loss of PM expression, as the constitutively activating V102A mutation restored conductance to both SS-Orail $\Delta \mathrm{C}_{273-301}$ and $\Delta \mathrm{N}_{1-77}$ OrailSS (Figure 2G). In view of the earlier demonstration that interaction of soluble S1C with the Orail NBD is essential for gating and that a similar NBD truncation significantly reduces this interaction in vitro [28], the lack of activation of $\Delta \mathrm{N}_{1-77}$ Orail by either co-expressed or C-terminally tethered S1C likely arises from a disruption of S1C binding to the truncated NBD. Similarly, loss of activation of $\Delta \mathrm{C}_{273-301}$ Orail by N-terminally tethered S1C likely arises from a disruption of S1C binding to the truncated CBD. Thus, our results show that directly linking S1C to Orail cannot rescue channel activation when critical regions of either the NBD or CBD of Orail are deleted. This suggests that S1C must interact with both the NBD and CBD to activate Orail.

\section{Hydrophobicity of residues 273 and 276 is required for channel activation}

The functional analysis of the CBD deletion mutants described above highlights the key role played by residues 272-280 in both binding to STIM1 and channel gating. These results are consistent with previous findings, which showed that removal of this region [44], or point mutation of leucines 273 and 276 (L273S/D and L276D) within this region [40, 42, 48], or combined mutations of several aspartate and glutamate residues within and close to this region (E272/275/278Q and D284/287/291N) resulted in loss of STIM1-dependent Orai1 channel activity $[49,50]$. Recently, an NMR structure of a C-terminal region of STIM1 containing parts of $\mathrm{CC} 1$ and $\mathrm{CC} 2$ domains $\left(\mathrm{CAD}_{312-387}\right)$ bound to Orail $\mathrm{CBD}$ $\left(\mathrm{CBD}_{272-292}\right)$ suggested that both polar and non-polar forces promote STIM1-CBD interaction and affect channel activation [46]. The SS-Orail construct offers a powerful tool to determine whether both types of interactions are required for channel gating when STIM1 is permanently anchored to Orail by an artificial link. We therefore removed all of the previously implicated CBD aspartates and glutamates by truncation and by alanine substitutions and found that this SS-Orail mutant remains functional, albeit to a reduced level (Figure 3A). These results suggest that electrostatic interactions between the acidic residues in the $\mathrm{CBD}$ and basic residues in CAD likely stabilize CAD-Orail interaction but are dispensable for channel gating. In contrast, we found that the single point mutation L273R or L273D in either SS-Orail or Orai1-SS eliminated channel activity. None of our point mutations of L276 abolished channel activity. Interestingly, however, while mutants L273S or L276D in both SS-Orai1 and Orai1-SS had current densities that were mildly reduced compared to WT, the double point mutation L273S/L276D eliminated channel activity in both SS-Orai1 and Orai1-SS contexts (Figure 3B), suggesting that these leucines contribute in a cooperative manner to activate the channel. To evaluate the significance of hydrophobic interactions between leucines 273/276 and S1C, we analyzed the side chain dependence at these positions for channel activation. We found that current density correlated with side chain hydrophobicity at both sites, although position 273 displayed stronger sensitivity to mutations that introduce polar or charged side chains (Figure 3C). As an alternative way to test whether the introduction of a charge at position 273 or 276 would interfere with channel activation, we analyzed the sensitivity of L273C and L276C mutants for application of 2-aminoethyl methanethiosulphonate (MTSEA), a positively charged cysteine-reactive reagent. Currents measured from cells expressing L273S, S263C or WT SSOrail were partially inhibited upon MTSEA application; however, this inhibition was fully reversed following washout of the reagent (Figure 3D and Supplementary information, Figure S4). Similar effects have been reported for WT Orail and for a cysteine-less Orail mutant, indicating that reversible MTSEA inhibition arises from non-covalent effects on ion conduction $[31,36]$. In contrast, L23C SS-Orai1 was strongly inhibited $(81.5 \%$ $\pm 3.9 \%$ ) and L276C SS-Orail was moderately inhibited $(44.4 \% \pm 10.7 \%)$, and the inhibition of these mutants was not reversed by MTSEA washout (Figure 3D-E). The inhibition was partially relieved by subsequent application of a reducing reagent (dithiothreitol or bis(2-mercaptoethylsulfone)) (Figure 2D and Supplementary information, Figure S4). These results indicate that channel inhibition by MTSEA is due to covalent modification of L273C and L276C, and support the interpretation that hydrophobic interactions at leucines 273/276 are critical for the activation of Orail channels. Together, these results indicate that S1C-CBD hydrophobic interaction plays a direct role in channel gating.

\section{Mutations in the Orail TM4-CBD linker abolish activa-} tion but not interaction with SIC

The dOrai structure shows that a conserved linker (Figure 1A and 4A) connects TM4 to the CBD helix, which projects into the cytosol [38]. A recent study found that the linker is not necessary for CAD-CBD interaction 
[46], but whether this region plays a role in channel activation is not known. To study its function, we initially attempted to interfere with linker geometry by substituting each individual residue with proline. We used the SS-Orail construct to ensure a high local concentration of S1C, while avoiding possible geometric constraints introduced by the proline mutations that may prevent a C-terminally tethered S1C from reaching the NBD. Proline substitution at positions 264 or 265 modestly reduced current density, while substitution at 266 strongly diminished current density and substitution at S263 abolished the current (Figure 4B). When co-expressed with untethered S1C, Orai1 S263P or Orai1 T266P did not produce detectible currents. Nuclear translocation of NFAT in cells co-expressing Orail S263P and S1C was similar to control (cells expressing Orail alone) (Figure 4C). Cells co-expressing Orai1 T266P and S1C, however, showed a mild increase in nuclear NFAT levels,
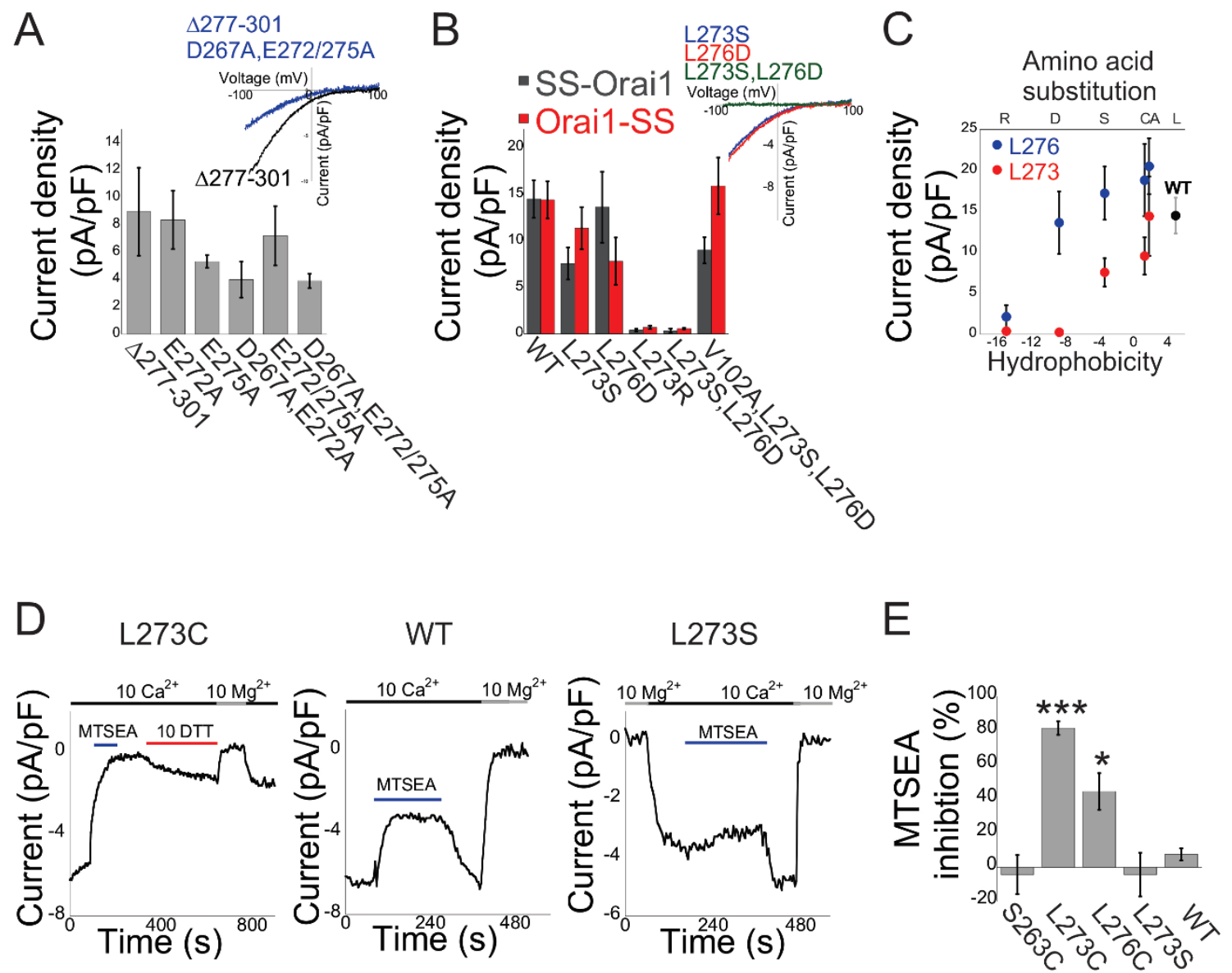

Figure 3 Leucines 273 and 276 compose a hydrophobic core in Orai1 CBD required for channel activation. (A) Summary of current densities recorded from cells expressing SS-Orai1 $\Delta \mathrm{C}_{277-301}$ with the indicated single, double or triple mutations $(n=$ 3-6 cells). Right, representative plots of the current-voltage relationship of currents recorded from cells expressing mCherrySS-Orai1 $\Delta \mathrm{C}_{277-301}$ or mCherry-SS-Orai1 $\Delta \mathrm{C}_{277-301}$ carrying the combination of D267A/E272A/E275A mutations. (B) Left, summary of current densities recorded from cells expressing mCherry-SS-Orai1 or Orai1-SS-EGFP with the indicated single, double or triple mutations ( $n=5-9$ cells). Right, representative plots of the current-voltage relationship of currents recorded from cells expressing the indicated mCherry-SS-Orai1 mutants. (C) Summary of current densities recorded from cells expressing mCherry-SS-Orai1 or Orai1-SS-EGFP with the indicated mutations plotted against the solvation energies of the substituted amino acids (free energies of transfer of amino-acid side chains to water from cyclohexane in kcal/mol [67]) ( $n=5-9 \mathrm{cells}$ ). (D) Time course of current modification by MTSEA $(500 \mu \mathrm{M})$ in cells expressing the indicated mCherry-SS-Orai 1 mutants. Note that significant persistent inhibition is seen only for mCherry-SS-Orai1 L273C following washout of MTSEA that is partially reversed by DTT (10 mM). (E) Summary of MTSEA-induced inhibition measured in cells expressing the indicated mCherry-SSOrai1 mutants ( $n=3-7$ cells). MTSEA-induced inhibition is quantified by normalizing currents measured post washout of the reagent to currents measured prior to reagent application. DTT, dithiothreitol. 
suggesting that low level of channel activation was preserved in this mutant (Figure 4C). Conductance of SSOrai1 S263P was restored by the constitutively activating V102A mutation (Figure 4G), indicating that the S263P mutation affected activation by S1C, but not protein folding, PM expression, channel assembly or the ability to conduct ions. To test whether flexibility/stability of the linker region affects Orai1 channel activation by STIM1, we replaced the first three residues in the linker with glycines (Orai1 3G) to create a flexible linker or with alanines (Orail 3A) to restrict the number of allowed conformations of the linker. Similar to S263P, when co-expressed with S1C, the Orai1 3G mutant did not produce detectible currents or nuclear translocation of NFAT (Figure 4C and 4D). In contrast, the Orai1 3A mutant exhibited moderate levels of channel activation (Figure 4C and 4D). These results suggest that linker flexibility/ stability affects the coupling between S1C-Orai1 interaction and channel gating. To further examine the effect of the linker region on functional coupling between S1C and Orai1, we studied the recently reported regulation of ion selectivity of Orail channel by STIM1. CRAC channels have the remarkable property that STIM1 binding not only markedly increases the activity of the channel but also alters its ion selectivity. This feature is particularly noticeable in constitutively conducting V102 mutants, including V102A, which forms a non-selective $\mathrm{Ca}^{2+}$ channel on its own, but becomes $\mathrm{Ca}^{2+}$ selective following interaction with STIM1 [31]. We analyzed the ion selectivity of Orail and SS-Orail V102A mutants by quantifying the reversal potentials of mutant channel currents in a calcium-containing solution. In agreement with earlier studies [28, 31], we found that V102A Orail mediates a non-selective constant cation current when expressed alone $\left(V_{\text {rev }}=19.6 \pm 5.2 \mathrm{mV}\right)$, while co-expression of the V102A mutant channel with STIM1 produced a rightward shift in current reversal potential $\left(V_{\text {rev }}=45.5\right.$ $\pm 5.6 \mathrm{mV}$ ), indicating that the interaction with STIM1 affects its ion selectivity (Figure 4E and 4G). A similar rightward shift in current reversal potential was recorded for cells expressing SS-Orai1 V102A $\left(V_{\text {rev }}=43.9 \pm 6.5\right.$, Figure 4F and 4G). However, in the SS-Orai1 V102A/ S263P double mutant, the reversal potential was similar to what is seen in V102A mutants without STIM1 ( $V_{\mathrm{rev}}=$ $12.1 \pm 5.5$, Figure $4 \mathrm{~F}$ and $4 \mathrm{G}$ ), indicating that the S263P mutation prevents the tethered S1C from modulating ion selectivity. To gain further insight into the mechanism through which the T266P, S263P and 3G mutations disrupt gating, we asked whether S1C binding to these mutant Orail's was also eliminated. Co-localization and FRET analyses revealed that compared with wt Orail the T266P, S263P and 3G mutants each retained signif- icant, although reduced, degree of interaction with S1C (Figure 5A-5D and Supplementary information, Figure S5). In addition, following ER $\mathrm{Ca}^{2+}$ depletion with $2 \mu \mathrm{M}$ thapsigargin, an irreversible blocker of SERCA (sarcoplasmic/ER Ca2+-ATPase), one could detect co-localized punctate fluorescence signals from STIM1-GFP co-expressed with mCherry-Orai1 S263P or mCherry-Orail $3 \mathrm{G}$ at the PM, consistent with the preservation of physical association of these Orail mutants with full-length STIM1 (Figure 5A). The interaction between S1C and Orail was completely lost when the S263P mutation was combined with L273S (Orai1 S263P/L273S), indicating that the CBD remained critical for S1C-Orail interaction (Figure 5D). The findings that mutations in the linker region abolish two functional effects of S1C, channel opening and modulation of ion selectivity, without eliminating interaction with the channel suggest that the Orail CBD linker region plays a critical role in coupling S1C binding to channel activation.

\section{Mutations at a TM4 proline bend cause STIM1-indepen- dent constitutive Orail activation}

The importance of the TM4-CBD linker for coupling STIM1-Orai1 binding with channel gating led us to ask whether the gating signal is transduced through TM4 of Orai1. The crystal structure of dOrai drew our attention to a conserved proline that forms a bend at the middle of TM4 (Proline 245 in hOrai1, Figure 1A and 4A). We hypothesized that if STIM1 binding to the Orail CBD induced a rearrangement in TM4, then this structural feature of TM4 could play an important role in gating. Consistent with this hypothesis, substituting P245 with alanine or leucine, which raises helical propensity, generated spontaneous current with rectifying current-voltage relationship and sensitivity to $\mathrm{La}^{3+}$ in the absence of heterologously expressed STIM1 (Figure 6A and Supplementary information, Figure S6). Measurements of current density and reversal potential indicated that the Orai1 $\mathrm{P} 245 \mathrm{~L}$ mutant exhibits reduced current density $\left(\underline{I}_{\mathrm{P} 245 \mathrm{~L}}=5.25 \pm 0.96 \mathrm{pA} / \mathrm{pF}, I_{\mathrm{CRAC}}=13.2 \pm 3 \mathrm{pA} /\right.$ $\mathrm{pF})$ as well as reduced $\mathrm{Ca}^{2+}$ selectivity compared to STIM1-activated wt Orail (Orail P245L $V_{\text {Rev }}=37.7 \pm$ $6.7 \mathrm{mV}$, STIM1-Orai1 $V_{\text {Rev }}=69.5 \pm 4.4 \mathrm{mV}$, in $10 \mathrm{mM}$ $\left.\mathrm{Ca}^{2+}\right)$. This finding could potentially be explained if the P245L mutation resulted in partial activation by binding of STIM1 to a fraction of its binding sites on Orail [31, 45]. Alternatively, the $\mathrm{P} 245 \mathrm{~L}$ mutation might activate the channel in a STIM1-independent manner. To address the nature of the activated state of Orail P245L, we initially asked whether increased expression of STIM1 could produce full activation and restore full $\mathrm{Ca}^{2+}$ selectivity to $\mathrm{P} 245 \mathrm{~L}$ Orai1 channels. In cells co-expressing Orai1 
A

H. sapiens
M.musculus
X. laevis
D.rerio
D.melanogaster
C. intestinalis
C.elegans

TM4

241 IMVPFGLIFIVFAVHFYRSLVSHKTDROFQELNELAE 279

244 IMVPCGLVFIVFAVHFYRSLVSHKTDRQFQELNELAE 282

199 IMVPFGLVFIVFAVHFYRSLVSHKTDRQFQELNELAE 236

163 IMVPFGLVFIVFAVHFYRSLVSHKTDRQFQELEELED 200

284 VLIPVMIIFMAFAIHFYRSLVSHKYEVTVSGIRELEM 321

215 VLIPVVILFAGFAVHFYRTLAQHRYTIMEDAFNEIET 252

235 MLVPVGVVFVVFSYLIHKNRVSHSLGRFKHKVDTMKQ 272

4

TM4 bend $\boldsymbol{4}$

TM4-CBD

linker

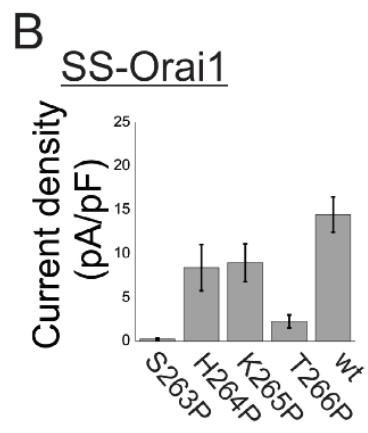

C

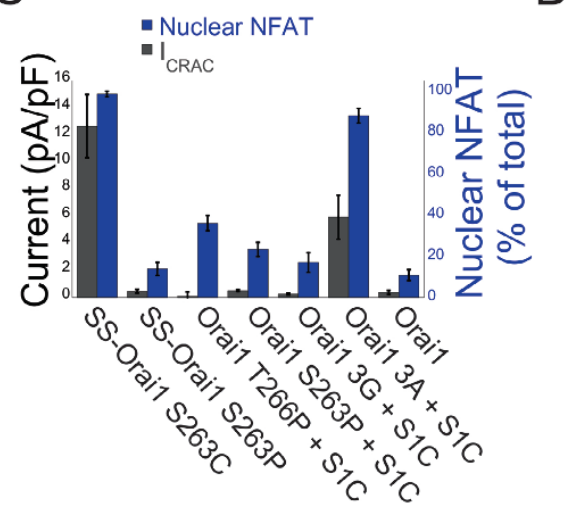

D

Orai1 $3 X+$ S1C
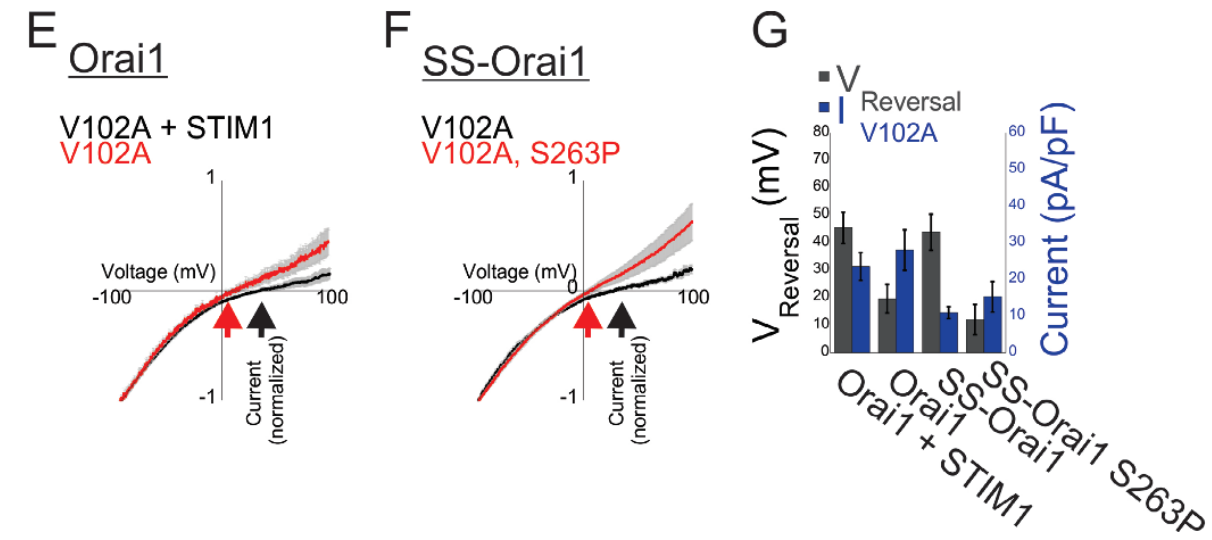

Figure 4 Mutations in the Orai1 C-terminal linker region eliminates channel activation and regulation of ion selectivity by S1C. (A) Amino-acid sequence alignment of TM4 (cyan background), linker (white background) and CBD (green background) regions from the indicated Orai1 homologs. Positions of mutated residues studied in this work are in highlighted in red. (B-D) Orai1 linker region mutations S263P and T266P abolish and diminish channel activation, respectively. (B) Summary of current densities recorded from cells expressing SS-Orai1 with the indicated proline substitutions $(n=3-7$ cells). (C) Summary of current densities ( $n=3-7$ cells) and quantification of nuclear localization of NFAT-GFP in HEK293 cells transfected with the indicated mCherry-SS-Orai1 or mCherry-Orai1 constructs alone or co-transfected with mCherry-S1C ( $n=95-196$ cells). (D) Representative plots of the current-voltage relationship of currents recorded from cells co-expressing the indicated mCherry-Orai1 mutants together with EGFP-S1C. (E, F) Normalized plots of current-voltage relationship of currents recorded in 10 $\mathrm{mM} \mathrm{Ca}{ }^{2+}$ Ringer's solution from cells expressing the indicated mCherry-Orai1 mutant alone or together with STIM1 (E) or from cells expressing the indicated mCherry-SS-Orai1 mutants (F). Arrows indicate the reversal potential in each instance. (G) Summary of reversal potentials and current densities recorded from cells expressing the indicated Orai1 or SS-Orai1 mutants ( $n=5-9$ cells $)$. 
P245L and STIM1, but not wt Orai1 and STIM1, we recorded spontaneous current immediately after cell breakin (Supplementary information, Figure S7). However, following passive $\mathrm{Ca}^{2+}$ store depletion by dilution of the cytosol into the patch pipette, current density and $\mathrm{Ca}^{2+}$ selectivity were indistinguishable between cells co-expressing Orai1 P245L and STIM1 and cells co-expressing wt Orail and STIM1 (Supplementary information, Figure S7), indicating that P245L Orail channels are partially activated prior to the turn-on by STIM1 and that STIM1 activation maximally activates the P245L mutant channels. Several lines of evidence indicated that the spontaneous activation of $\mathrm{P} 245 \mathrm{~L}$ and $\mathrm{P} 245 \mathrm{~A}$ mutant channels was independent of endogenous STIM1 and STIM2. Constitutive current was recorded in cells expressing Orai1 P245L or P245A alone, but not WT Orai1 alone (Figure 6A and Supplementary information, Figure S6). Similarly, $\mathrm{Ca}^{2+}$ imaging and NFAT translocation assays revealed constitutive $\mathrm{Ca}^{2+}$ entry in cells expressing Orai1 P245L or P245A alone, but not WT Orail alone (Figure 6D, 6E and Supplementary information, Figure S6). Importantly, knockdown of endogenous STIM1

A
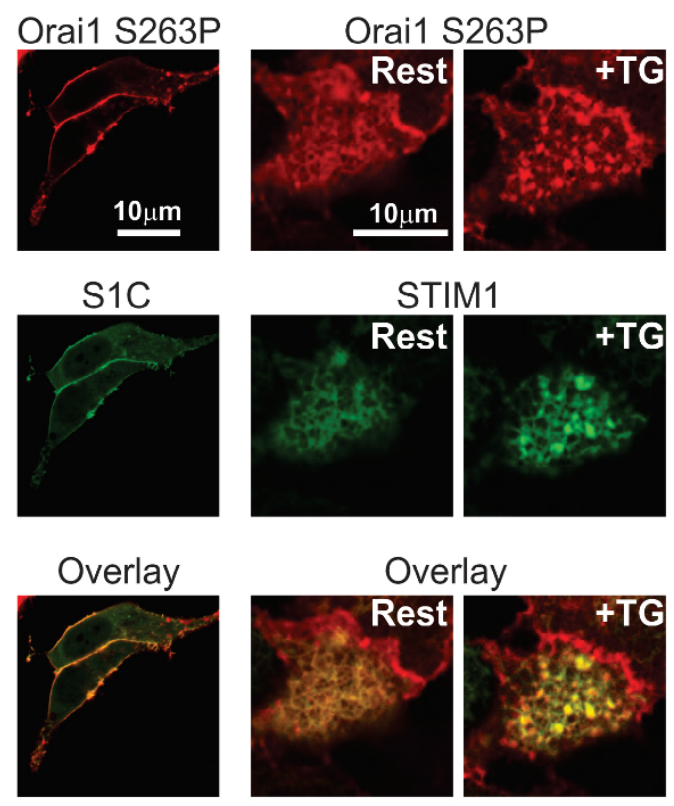

Overlay
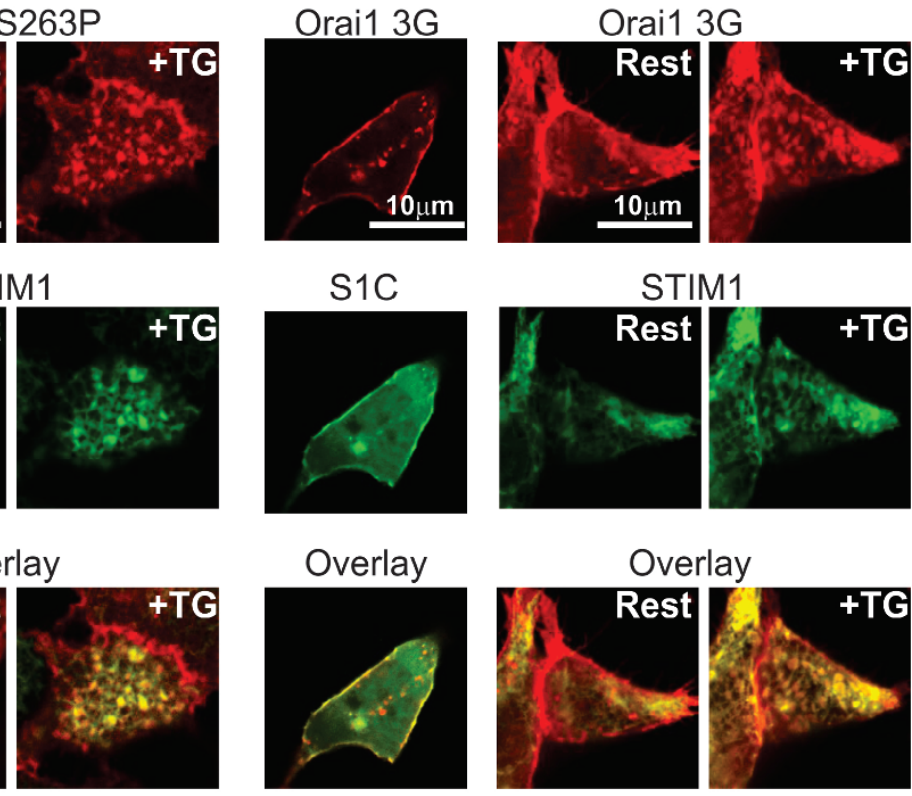

STIM1
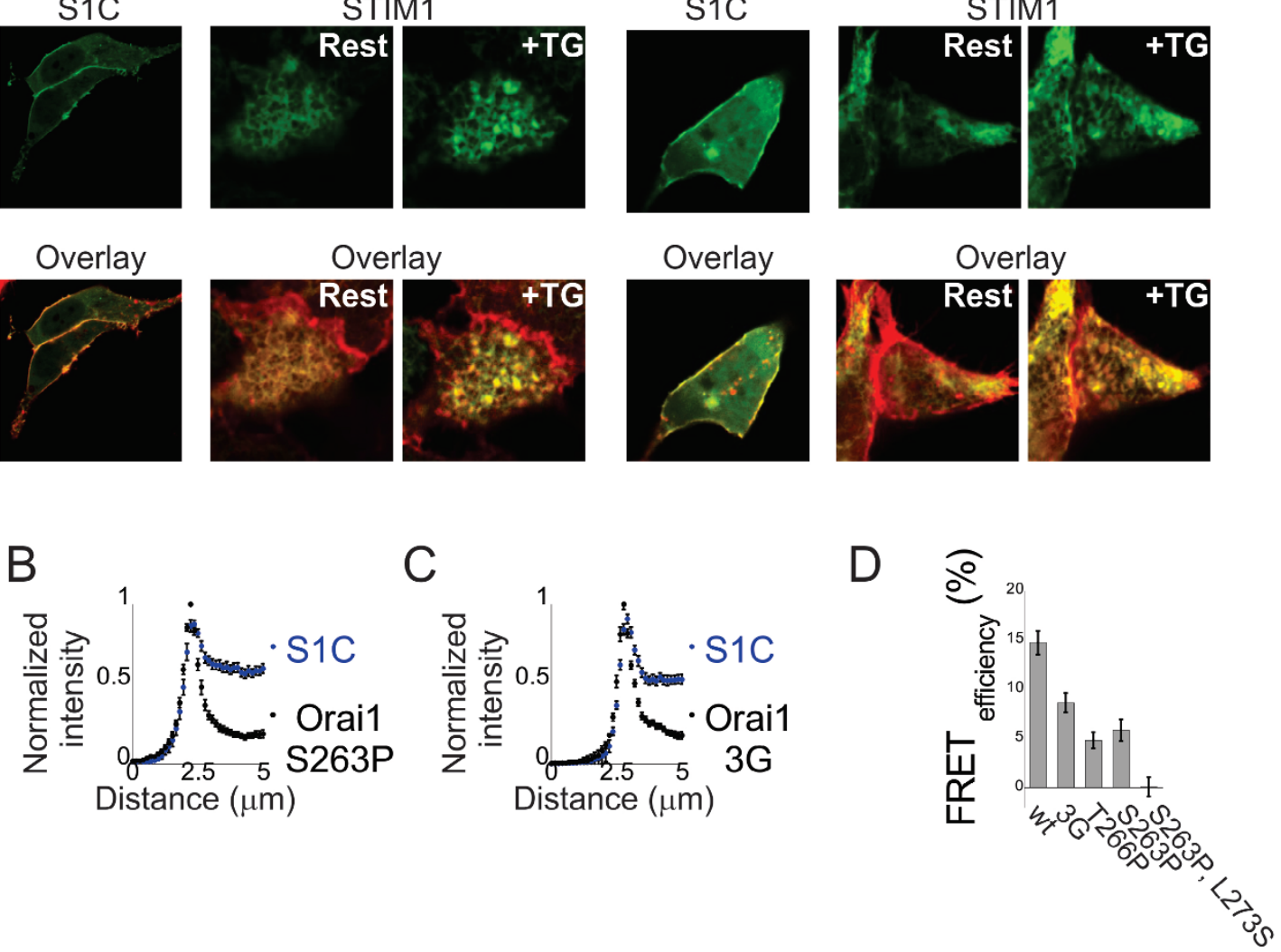

Figure 5 STIM1 interacts with the non-functional Orai1 CBD-TM4 linker mutants. (A) Representative fluorescent images of cells expressing EGFP-S1C or STIM1-EGFP together with the indicated mCherry-Orai1 mutant before $\left(2 \mathrm{mM} \mathrm{Ca}^{2+}\right)$ and after application of TG $\left(2 \mu \mathrm{M}\right.$ TG, $\mathrm{Ca}^{2+}$ free). (B, C) Summary of normalized intensity values across the plasma membrane and intracellular regions of cells expressing EGFP-S1C together with indicated mCherry-Orai1 mutant ( $n=13-24$ cells). (D) Summary of FRET efficiency values measured in cells expressing EGFP-S1C together with mCherry-Orai1 or with the indicated mutants ( $n=15-19$ cells). TG, thapsigargin. 
A

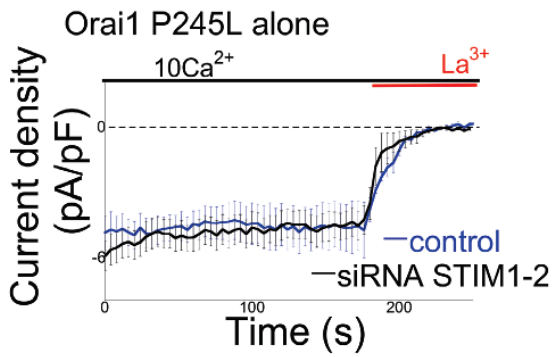

B

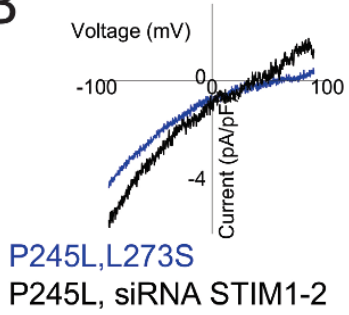

C

mCherry-Orai1 P245L alone

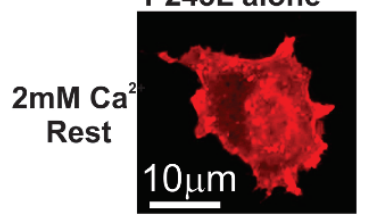

mCherry-Orai1 P245L

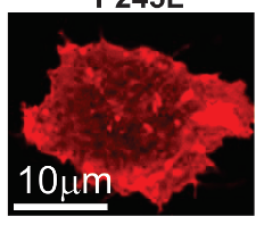

STIM1-EGFP

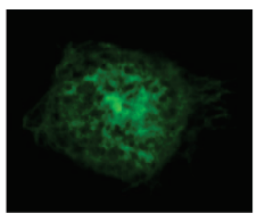

\section{Overlay}
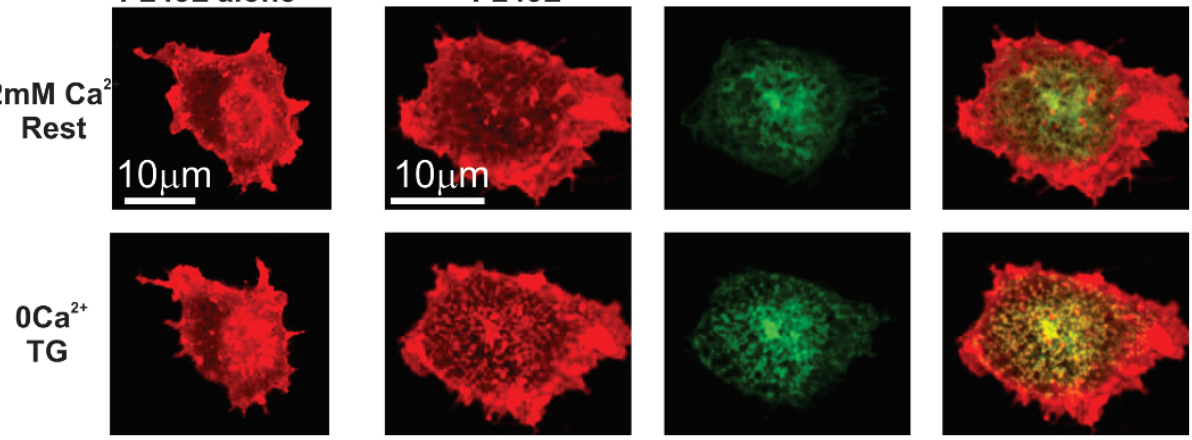

E
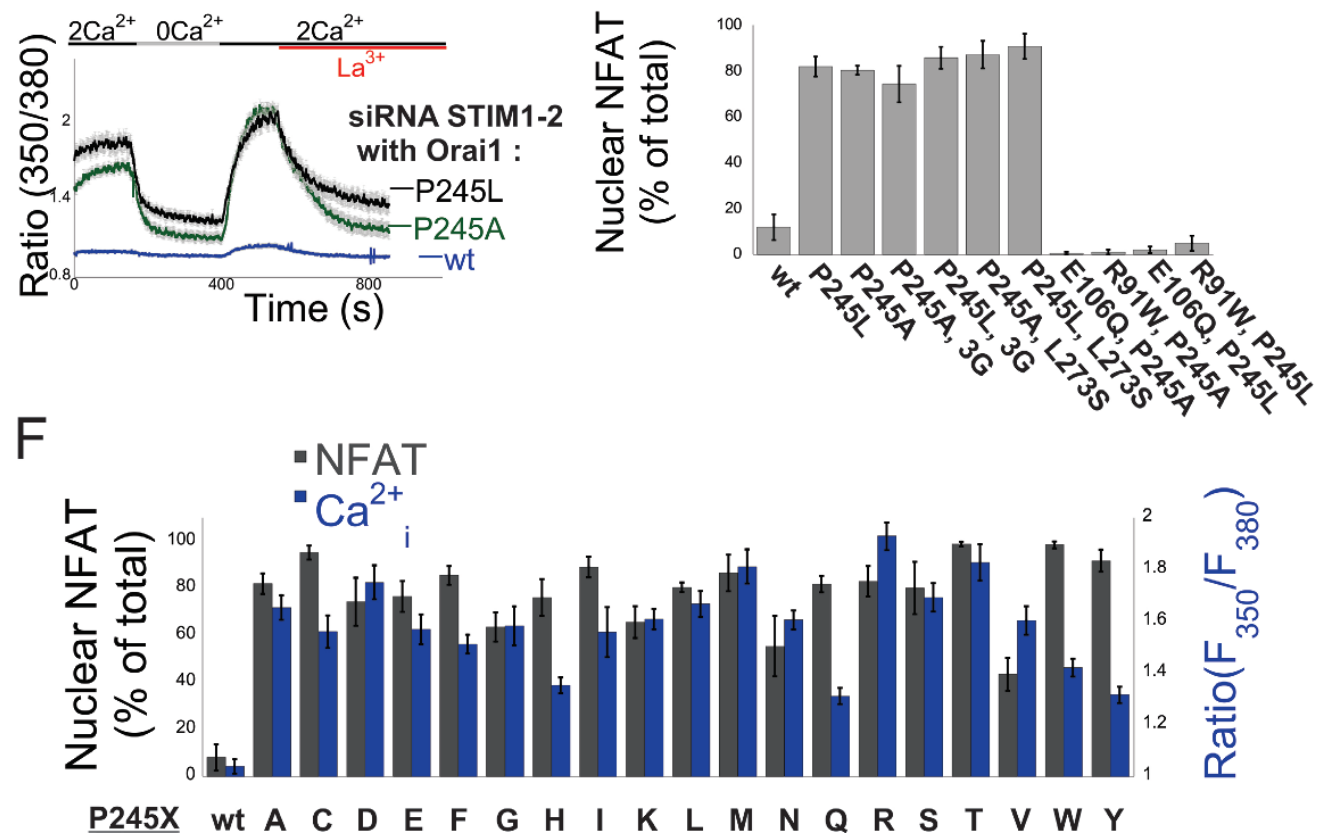

Figure 6 Mutations at TM4 proline bend cause STIM1-independent constitutive Orai1 activation. (A) Time course of spontaneous currents measured from untreated cells (control) or from cells pre-treated with siRNA against STIM1 and STIM2 following transfection with Orai1 P245L alone. (B) Representative plots of the current-voltage relationship of currents recorded from cells expressing the indicated mCherry-Orai1 mutants. (C) Representative fluorescent images of cells expressing mCherry-Orai1 P245L alone or together with STIM1-EGFP before $\left(2 \mathrm{mM} \mathrm{Ca}^{2+}\right)$ and after application of TG $\left(2 \mu \mathrm{M} \mathrm{TG}, \mathrm{Ca}{ }^{2+}\right.$ free). (D) Time course of intracellualr $\mathrm{Ca}^{2+}$ concentrations measured from cells pre-treated with siRNA against STIM1 and STIM2 and transfected with Orai1 or the indicated Orai1 P245 mutant alone. (E, F) Basal Ca ${ }^{2+}$ levels $(n=23-40$ cells) and quantification of nuclear localization of NFAT-GFP ( $n=47-240$ cells) in cells transfected with mCherry-Orai1 containing the indicated P245 mutation. TG, thapsigargin. 
and STIM2 did not affect the constitutive $\mathrm{Ca}^{2+}$ entry in cells expressing Orai1 P245L or P245A alone (Figure 6A, 6B, 6D and Supplementary information, Figure S6), although it strongly diminished store-operated calcium entry in cells expressing WT Orail alone (Supplementary information, Figure S8). Furthermore, analysis of puncta formation showed that, following $\mathrm{ER} \mathrm{Ca}^{2+}$ depletion with thapsigargin, Orai1 P245L remained diffusely distributed in the PM when expressed alone, but was recruited into puncta when co-expressed with STIM1 (Figure 6C). Along similar lines, current recorded from cells expressing Orai1 P245L alone did not exhibit fast $\mathrm{Ca}^{2+}$-dependent inactivation (CDI), but fast CDI was apparent when STIM1 was co-expressed with Orai1 P245L (Supplementary information, Figure S6). Thus, assays of STIM1-dependent modification of Orail distribution or function indicated that Orail P245L does not engage endogenous STIM proteins, even though it is constitutively active. In further confirmation of this interpretation, introducing the L273S mutation, which prevents STIM1 from binding to Orai1, together with P245L did not affect spontaneous NFAT nuclear translocation or current (Figure 6B, 6E and Supplementary information, Figure S6). Since P245, in the middle of TM4, is spatially remote from the pore-lining TM1 helix, we also considered the possibility that the mutations at P245 introduced a novel permeation pathway. To address this possibility, the pore mutation R91W or E106Q was introduced together with $\mathrm{P} 245 \mathrm{~L}$ or P245A. The combined mutations of P245L or P245A with R91W or E106Q in Orail completely abolished channel activity (Figure 6E), indicating that mutants P245L and P245A have the wild-type $\mathrm{Ca}^{2+}$ conduction pathway. We asked whether other mutations at this position would have the same effect and substituted P245 with each of the remaining amino acids. Strikingly, increased levels of intracellular $\mathrm{Ca}^{2+}$ and nuclear translocation of NFAT were observed under resting conditions in every one of the P245X mutants (Figure 6F). The observation that all mutations at the proline bend in TM4 induce an activated state of Orail channels in a STIM1-independent manner suggests that the natural bend at the wildtype proline is important for stabilizing channel closure and that opening occurs by STIM1-induced rearrangement that overcomes this locking mechanism.

\section{Discussion}

Orail $N B D$ and $C B D$ are both required for channel activation

Structural perturbations in the Orail NBD have been shown to disrupt Orail channel activation by STIM1, even if physical interaction between STIM1 and Orai1 is preserved, whereas CBD perturbations have been shown to disrupt both physical and functional interaction [37, 39-42]. These observations, along with biochemical evidence for direct binary interactions of STIM1's CAD with either the Orail NBD or CBD, and the discovery that the TM1 segment of Orail lines the pore and contains the gate, led to the Anchoring Model, in which the role of the Orail CBD is to anchor STIM1 via high-affinity binding and position it near the low-affinity NBD whose binding then triggers gating (Figure 1). This model predicts that the CBD could be replaced by an alternative method of physically linking STIM1 to Orail. In support of this notion, Zheng et al. [43] recently found that a CBD truncation mutant missing amino acids 277-301 (Orai1 $\Delta \mathrm{C}_{277-301}$ ), which was not activated by full-length STIM1, had activation restored when CAD was tethered to the truncated $\mathrm{C}$ terminal. However, whether soluble CAD could bind to and activate Orail $\Delta \mathrm{C}_{277-301}$ was not quantitatively or functionally tested in that study. In contrast, and arguing against the CBD anchoring model, McNally et al. [44] found that a different CBD truncation mutant, which was missing amino acids 272-279 (Orail $\Delta \mathrm{C}_{272-279}$ ), was not activated by $\mathrm{CAD}$, even when $\mathrm{CAD}$ was tethered to the truncated $\mathrm{C}$ terminus. In light of the crystal structure of dOrail, however, the concern arises that the inability of the linked CAD to activate the channel could reflect a geometric constraint due to the proximity of the CAD linkage site in this deletion construct, which could have prevented the CAD from reaching the NBD. Indeed, a change in the linker that connects the CAD to the CBD of Orail has been shown to affect the degree of channel activation [45]. Our results resolve the apparent discrepancy between these recent reports and argue against the CBD Anchoring Model. First, we show that an Orail CBD deletion mutant missing amino acids 277-301 retains physical and functional interaction with soluble S1C, but, and in agreement with the findings by Zheng et al. [43], activation of this mutant by STIM1 is strongly diminished (Supplementary information, Figure S2), likely because of the stronger binding of Orail by S1C than by full-length STIM1, as previously shown [14, 15]. Second, by tethering our S1C pair to the full-length $\mathrm{N}$ or $\mathrm{C}$ terminal, while mutating the other terminal, we could avoid the potential problem of steric hindrance. In this context, our finding that $\mathrm{CBD}$ deletion $\mathrm{C}$ terminally of leucine 273 abolishes channel activation shows that the CBD is more than just a STIM1-anchoring site. Third, as discussed in further detail below, C-terminal membrane and membrane proximal regions "above" the CBD are capable of modulating STIM1-dependent and STIM1-independent effects on the channel pore, thereby suggesting that regulation of pore opening could also be 
transduced by the CBD.

\section{A hydrophobic core in the membrane-proximal Orail $C B D$ is essential for channel gating}

The functional outcome of STIM1 interaction with Orai1 L273S/D and L276D mutants has been widely discussed in the literature. All studies that employed these Orail mutants found that they lacked physical association with STIM1. Consequently, these studies were unable to directly address the potential role of L273 or L276 downstream of STIM1-Orai binding, i.e., in gating. Recent reports [43-45], and results presented here, find that tethering S1C to Orail restores activation to some Orai1 mutants with single amino-acid substitutions or deletions in the CBD (L273S, L276D and $\Delta$ C276-301) that otherwise could not be activated by soluble S1C. These findings could suggest that L273 and L276 are important for facilitating STIM1-Orai1 association but not for channel gating. One study [45], however, found that L273D inhibits channel activation even in the context of Orail-SS, but the cause for this effect was not clarified. Phylogentic analysis of Orail homologs shows that conservation of residues at positions 273 and 276 is better characterized by residue hydrophobicity than by Leu identity (Figure 4A). Here we find that directly linking S1C to Orail fails to activate several CBD mutants (L273D, L273R and L273S/L276D) and does not restore full-channel activation to others (L273S, L273C and L276R). Analysis of these effects shows strong dependence of current density on side-chain hydrophobicity at position 273 , and to a lesser extent position 276. Moreover, complementary experiments employing reversible chemical modification suggest that introducing charge at either position strongly inhibits channel activity. Together, these findings demonstrate that hydrophobic interactions at L273 and L276 are critical for both binding of STIM1 and channel gating.

\section{TM4-CBD linker couples STIM1 binding with channel} activation

Residues L273 and L276, described above, are also interesting because in a resting state (STIM1 not bound) crystal structure of the Drosophila Orai they form a hydrophobic patch that connects the CBDs of neighboring subunits [38], while in an NMR structure of the human STIM1 CAD and Orail CBD complex, the resting state CBD-CBD interaction and the intra-CAD coiled-coil interaction between $\mathrm{CC} 2$ and $\mathrm{CC} 3$ of the crystal structure [46] are replaced by another hydrophobic interaction, this time between the CC2 of CAD and L273/L276 of the Orail CBD. Thus, the dependence of STIM1-Orai1 protein association and channel activation on L273 and L276 can be attributed to replacing the resting CBD-
CBD complex with the activated CAD-CBD complex. The CAD-CBD NMR structure also reveals that CADCBD interaction occurs in the absence of the TM4-CBD linker region. In agreement with this observation, we find that mutations to the TM4-CBD linker region only induce moderate effects on S1C binding while exerting marked effects on channel activation. The simplest interpretation of these results is that the TM4-CBD linker region is important for coupling CAD binding with Orail channel activation. Interestingly, however, the moderate effects of the TM4-CBD linker region mutations on S1COrail interaction raise the possibility that the TM4-CBD linker region is also important for precise positioning of the CBD that may be required for optimum CAD binding. The finding that Orail gating is disrupted either by interference with formation of the CAD-NBD or CADCBD complex or by mutation to the TM4-CBD linker suggests that gating involves signal transmission of STIM1 binding at the cytoplasmic NBD to TM1 and at the CBD to TM4 through the TM4-CBD linker.

\section{TM4 regulates Orail gating}

An indication of coupling between STIM1 binding at the CBD and the channel gate was obtained recently from the effect of AnCoA4, a new Orail channel inhibitor, which binds only to the $\mathrm{C}$ terminus of Orail and inhibits channels made constitutively active by the TM1 mutation V102C [51]. But how is CAD-CBD interaction transmitted to the channel gate? Our finding that mutations that are expected to disrupt the P245 proline bend of TM4 open the channel suggests that the rearrangement of TM1 that has been proposed to underlie gating [31, $52,53]$ may be triggered by a conformational change in TM4. We do not know whether mutations to P245 induce a conformational change in Orail that is similar to that induced by STIM1. However, the finding that STIM1 binding to P245L Orai1 maximally activates the channel indicates that the mechanism that couples STIM1 binding with pore opening is preserved. Strikingly, the P245 bend of TM4 is situated in the same membrane plane as residues in each of the other helices (Supplementary information, Figure S9) that have been implicated in gating: residue G98 in TM1, which is thought to pivot to open the pore [53], residue H134 in TM2 whose mutation constitutively open the channel independently of STIM1 [54] and residue G183 in TM3, whose mutation prevents STIM1-dependent channel activation [55], but allows for channel opening by 2-APB, indicating a defect in STIM1 coupling but not in gating (Supplementary information, Figure S9).

We find that every amino acid other than proline at P245 induces some degree of STIM1-independent Orai1 
channel activation. In other words, only proline stabilizes the closed channel conformation. By forming hinges in transmembrane helices, proline residues were previously shown to act as switches that play important roles in the function of several ion channels including the 5-hydroxytryptamine type 3 receptor $[56,57]$ and voltage-dependent $\mathrm{K}^{+}$channels [58-61]. In view of the conformation of the closed state (STIM1 not associated) structure of dOrail, in which TM4 is bent at P245 (21), we suggest that this bend is crucial for the closed conformation and that the energy of CAD binding to both the NBD and CBD activates the channel by stabilizing a straight conformation of TM4.

How mutation in the TM4 proline bend might induce myopathy

The P245L mutation was recently associated with tubular aggregate myopathy and congenital miosis in humans, and the dysfunction attributed to a decrease in slow $\mathrm{Ca}^{2+}$-dependent inactivation [62]. It is worth noting that the electrophysiological analysis in that study, however, did detect small spontaneous currents $(1.5-2 \mathrm{pA} / \mathrm{pF}$ with maximal currents of $\sim 10 \mathrm{pA} / \mathrm{pF}$ ) upon cell break-in and before STIM1 activation consistent with our findings ( $2.5 \mathrm{pA} / \mathrm{pF}$ with maximal current of $13.4 \mathrm{pA} / \mathrm{pF}$, Supplementary information, Figure S7A and S7B). Recent studies had shown that slow $\mathrm{Ca}^{2+}$-dependent inactivation involves a concerted action of SARAF and STIM1 [63, 64] but whether Orail is directly involved in the process remains unknown. While it is possible that $\mathrm{P} 245 \mathrm{~L}$ Orai1 is less sensitive to slow $\mathrm{Ca}^{2+}$-dependent inactivation, another plausible scenario is that during slow inactivation, STIM1 activation of Orai1 P245L is gradually lost. This process leaves a "STIM1-free" but partially active Orai1 P245L that contributes to the measured current and therefore to an apparent reduction of the slow inactivation process. Two other mutants, G98S and L138F, which have also been associated with tubular aggregate myopathy and congenital miosis syndromes [65], share the defect that we identified in P245L of constitutive channel opening $[43,53,65]$. Although the constitutive conductance of Orail P245L may be less pronounced in heterozygous patients than in heterologous overexpression experiments due to formation of mutant and WT heteromultimers, based on results presented here and on previous findings, we propose that STIM1-independent constitutive channel activation of Orail channels is likely the common factor that underlies the disease state.

\section{A molecular mechanism for CRAC channel gating}

The results presented here, together with those from previous studies, provide evidence for a dual trigger
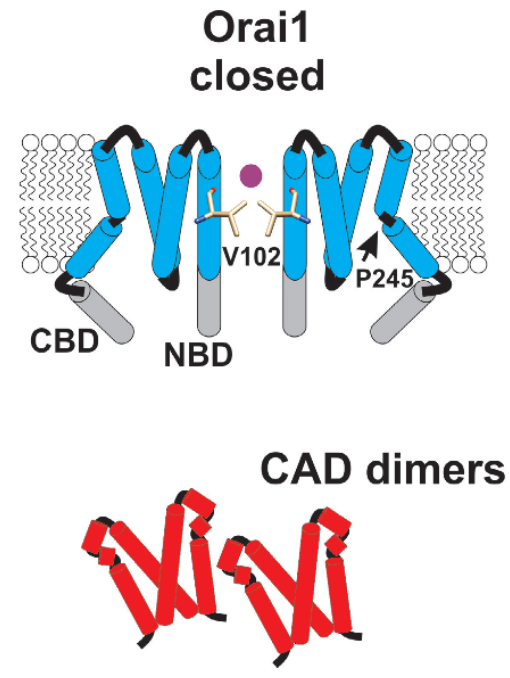

Orai1-CAD open

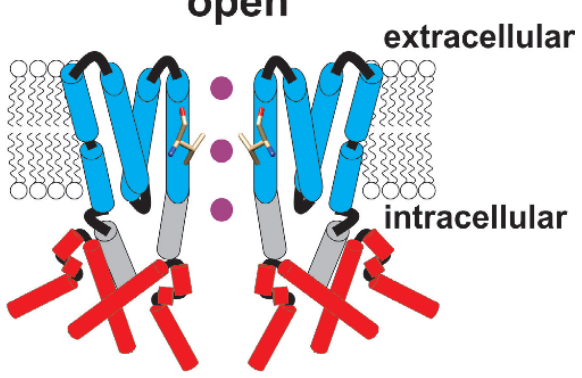

Figure 7 Hypothetical model for CRAC channel gating. Cartoon illustration of the closed (left) and open (right) states of Orai1 (transmembrane helices are represented by blue cylinders and the NBD and CBD extensions by grey cylinders) following interaction with CAD (red). (Left) The closed conformation of Orai1 channel. The TM4 bend restricts movement of TM2 and 3 and locks TM1 in place with the side chain of V102 projecting towards the pore to prevent ion permeation. (Right) The open state of Orai1 is induced by binding of CAD to the NBD and CBD of Orai1. CAD-binding pulls the CBD to induce a conformational change in the C-terminal region of Orai1 that modulates TM4. The straightening of TM4 is propagated through TM2TM3 to induce rearrangement of a region of the pore-lining TM1 helix which includes repositioning of the hydrophobic side chain of valine 102 away from the pore thereby allowing ion permeation. 
model of Orail channel opening. In this model (Figure 7), STIM1 binds to, and induces local conformational changes in, both the $\mathrm{N}$-terminal region and $\mathrm{C}$-terminal region of Orail. Recent studies identified determinants in the N-terminal region of Orai1 that communicate STIM1 binding to the NBD "up" into TM1, which lines the pore and contains the channel gate. We have identified determinants in the Orail C-terminal region that communicate STIM1 binding to the CBD "up" through the TM4-CBD linker and into TM4 to modulate TM4 at its proline bend (P245) and thereby activate the channel. The similar transmembrane position of the P245 proline bend in TM4 and gating residues in TM1, as well as coupling residues in TM2 and TM3 (Supplementary information, Figure S9), suggests that channel activation involves a synchronized helical rearrangement that is coordinated by the simultaneous allosteric effects of STIM1 binding at the two termini of Orail.

\section{Materials and Methods}

\section{Cell culture and transfection}

HEK293 cells were cultured in DMEM as previously described [64]. Plasmid transfection of cells was performed using Lipofectamine 2000 (Invitrogen) according to the manufacturer protocol. For electrophysiological experiments, 6-8 $\mathrm{h}$ after plasmid transfection and $12-15 \mathrm{~h}$ before start time of experiments, cells were plated onto 18-mm cover glass coated with L-polylysine. $\mathrm{For}^{2 a^{2+}}$ imaging, NFAT translocation, co-localization and FRET experiments, cells were plated onto 18-mm cover glass coated with L-polylysine and 6-8 $\mathrm{h}$ after plasmid transfection culture, media was replaced to wash the transfection reagent. To avoid constitutive calcium rise in cells expressing all forms of SS-Orai1, Orai1-SS, CAD or V102A mutants, cells were cultured in high glucose, $\mathrm{Ca}^{2+}$-free DMEM supplemented with $50 \mu \mathrm{M} \mathrm{La}^{3+}$.

For knock-down of endogenous STIM1 proteins, cells were transfected $\sim 72 \mathrm{~h}$ before the experiment with small interfering RNAs (siRNAs) and then re-transfected $\sim 24 \mathrm{~h}$ before the experiment with the indicate plasmid. The siRNAs used in this work were purchased from Sigma-Aldrich (MISSION Predesigned siRNA). The MISSION siRNA Universal Negative Control \#1 were used as control siRNAs and sequences for STIM1 and STIM2 siRNAs were:

STIM1 siRNA:

SASI_Hs01_00107804: 5'-GAGATTGTGTCTCCCTTGT-3' SASI Hs01 00107805: 5'-CTCACTTCATCATGACTGA-3' STIM2 SiRNA:

SASI Hs02 00354130: 5'-CTTCATTTATGATCTCCCA-3'

SASI_Hs02_00354131: 5'-GATCTGTGGCTTTCAGATA-3'.

\section{Electrophysiological recordings}

Membrane currents were recorded under voltage-clamp conditions using whole-cell patch-clamp configuration on an Axopatch 200B amplifier (Axon Instruments). Patch pipettes were fabricated from borosilicate glass capillaries (4-8 M $\Omega$ ). Signals were acquired at $100 \mathrm{KHz}$ and filtered using a $2 \mathrm{kHz}$ low-pass
Bessel filter. Data acquisition and analysis were performed using pCLAMP 10 software (Axon Instruments). Two voltage protocols were employed; the first consisted of a 100-ms ramp from -100 to $+100 \mathrm{mV}$ and the second consisted of $250 \mathrm{~ms}$ step to $-120 \mathrm{mV}$ followed by a 100 -ms ramp from -100 to $+100 \mathrm{mV}$ delivered every 1 or $4 \mathrm{~s}$ from a holding potential of $0 \mathrm{mV}$. Current densities were calculated by normalizing currents measured at $-90 \mathrm{mV}$ to cell capacitance. Internal solution contained $150 \mathrm{mM} \mathrm{Cs}$ aspartate, $8 \mathrm{mM} \mathrm{MgCl}_{2}, 10 \mathrm{mM}$ HEPES and $8 \mathrm{mM}$ BAPTA. To measure fast $\mathrm{Ca}^{2+}$-dependent inactivation $8 \mathrm{mM}$ BAPTA was replaced with 1.2 mM EGTA. CsOH was used for adjustment of $\mathrm{pH}(\mathrm{pH}=7.2)$. External Ringer's solution contains $145 \mathrm{mM} \mathrm{NaCl}, 2.8 \mathrm{mM} \mathrm{KCl}$, $10 \mathrm{mM}$ HEPES and $10 \mathrm{mM}$ glucose (pH 7.4 with $\mathrm{NaOH}) . \mathrm{CaCl}_{2}$ $(10 \mathrm{mM})$ or $10 \mathrm{mM} \mathrm{MgCl}$ was added to the external solution for high- $\mathrm{Ca}^{2+}$ or Ca-free solution, respectively. The Divalent-free (DVF) solution contained: $150 \mathrm{mM} \mathrm{NaCl}$ (DVF-Na) or $150 \mathrm{mM}$ CsCl (DVF-Cs), $10 \mathrm{mM}$ HEDTA, $1 \mathrm{mM}$ EDTA and $10 \mathrm{mM}$ Hepes ( $\mathrm{pH}$ 7.4). STIM1 activation was induced by passive store depletion and currents measured from cells co-expressing STIM1 and Orai1 normally reached maximum within 2-3 min from cell break-in. Whole-cell recordings in cells co-expressing STIM1 with mutant Orail channels were hence performed for a period of 4-10 min to ensure sufficient time for STIM-Orail activation. All data were leak corrected using the current elicited in high $\mathrm{Ca}^{2+}$ Ringer's solution supplemented with $10-100 \mu \mathrm{M} \mathrm{La}^{3+}$ or in $\mathrm{Ca}^{2+}$-free Ringer's solution, as appropriate.

\section{Measurements of intracellular calcium levels}

Transfected cells grown on glass coverslips were incubated for 30 min with $5 \mu \mathrm{m}$ Fura-2 AM (Molecular probes, Life Technologies) in standard Ringer's solution $(140 \mathrm{mM} \mathrm{NaCl}, 10 \mathrm{mM}$ HEPES, $10 \mathrm{mM}$ glucose, $0.8 \mathrm{mM} \mathrm{MgCl}, 2.8 \mathrm{mM} \mathrm{KCl}$ and $2 \mathrm{mM}$ $\mathrm{CaCl}_{2}$, at $\mathrm{pH}$ 7.4) and allowed to equilibrate for additional 5-10 min without dye. Following dye loading coverslips were mounted in a chamber and emission (510-nm long pass) images were collected during alternate excitation at $350 \pm 5$ and $380 \pm 5 \mathrm{~nm}$ using a filter wheel (Lambda-10, Sutter Instruments). The imaging system consisted of a Nikon Diaphot inverted microscope equipped with a $20 \times$ objective, and CCD camera (SenSys, Photometrics). Axon Imaging Workbench 4.0 (Axon Instruments) controlled both filters and collection of data.

\section{Co-localization analysis}

Cells co-expressing mCherry-Orai1 or various mCherry Orai1 mutants together with EYFP-S1C or EGFP-S1C (hSTIM1 fragment corresponding to residues 343-465) were plated and cultured as described above. Mid-planes sections of transfected cells were captured using LSM 780 confocal microscope (Zeiss) controlled by Zen imaging software (Zeiss). EGFP or EYFP were excited at $488 \mathrm{~nm}$ and emission collected from 493 to 524 (for EGFP) or from 493 to $540 \mathrm{~nm}$ (for EYFP), and mCherry was excited at 561 $\mathrm{nm}$ and emission collected from 583 to $685 \mathrm{~nm}$. The parameters used for image acquisition were kept constant across individual sets of experiments and analysis was restricted to cells with similar EYFP and mChery fluorescence to ensure similar ratios of S1C/ Orail expression. Line-scans of regions spanning the PM and cytosol were analyzed from individual images using Image-J. Each line had a total scan width of 36 pixels corresponding to a length of $5 \mu \mathrm{m}$. Fluorescent intensity along each individual scan was 
normalized to peak value. Finally, normalized line-scans of both mCherry-Orail and EYFP-S1C from individual cells were aligned according to the peak maximum of mCherry-Orail to produce cellular intensity plots.

\section{Acceptor photobleaching FRET}

Cells co-expressing mCherry-Orail or various mCherry Orai1 mutants together with EGFP-S1C (hSTIM1 fragment corresponding to residues 343-465) were plated and cultured as described above. Cells were fixed in $4 \%$ fresh paraformaldehyde for $30 \mathrm{~min}$ and washed three times for $10 \mathrm{~min}$ with PBS. Cells were subsequently imaged in PBS on a LSM 780 confocal microscope (Zeiss). The donor (EGFP) was excited at $488 \mathrm{~nm}$ and emission collected from 493 to $524 \mathrm{~nm}$, and the acceptor (mCherry) was excited at $561 \mathrm{~nm}$ and emission collected from 583 to $685 \mathrm{~nm}$. EGFP-S1C donor fluorescence was imaged before and after bleaching a region of interest of mCherry-Orai1. Following bleaching, mCherry-Orai1 fluorescence was decreased to $<10 \%$ of its initial intensity. FRET energy transfer $\left(\mathrm{FRET}_{\text {efficiency }}\right)$ was calculated as $\%\left(\mathrm{FRET}_{\text {efficiency }}\right)=$ $100 \times\left(D_{\text {post }}-D_{\text {pre }}\right) / D_{\text {post }}$, where $D_{\text {pre }}$ and $D_{\text {post }}$ are the donor (EGFP) intensities before and after acceptor (mcherry) bleaching, respectively.

\section{NFAT translocation assay}

Cells co-expressing the indicated Orai1 constructs together with NFAT-EGFP or NFAT-mCherry were plated and cultured as described above. At the start of each experiment, cells were washed twice with Ringer's solution containing $2 \mathrm{mM} \mathrm{Ca}^{2+}$ and kept in the same solution for $45 \mathrm{~min}$ after which mid-planes sections of transfected cells were acquired using LSM 780 confocal microscope (Zeiss). Images were analyzed using Image-J to determine cytosolic or nuclear localization of NFAT-GFP in individual cells.

\section{Generation of plasmids}

The STIM1-GFP construct was previously described [64] and S1C cDNA was PCR amplified from this construct and inserted into p3XFLAG 7.1 in frame with an existing cDNA coding for mCherry to create mCherry-S1C. EYFP-S1C and EGFP-S1C were created by insertion of S1C fragment from mCherry-S1C into EGFP-C1 or EYFP-C1 using BsrGI-BamHI sites. The mCherry-hOrail construct was obtained from Professor Shmuel Muallem's lab at the National Institutes of Health (NIH, USA). CC-terminal deletion mutants of mCherry-Orail were created by insertion of one or more consecutive stop codons at sites corresponding to positions 285, 281, 277, 276 and 273 in hOrai1. The mCherry-Orail construct was also used as template to create mCherry-S-Orai1 and mCerry-SS-Orai1. The first S1C (S module) cDNA was made by a standard PCR amplification after which it was used as a mega primer inserted into mCherry-Orail by a PCR-based and restriction-free cloning procedure [66] to create mCherry-S-Orai1. A second S1C cDNA was subsequently inserted in frame into mCherry-S-Orail using a Not I site. C-terminal deletion mutations of mCherry-SS-Orail were created by insertion of one or more consecutive stop codons at sites corresponding to positions 285, 281, 277, 276 and 273 in hOrai1. The Orail-SSEGFP construct was obtained from Professor Tao Xu's lab at the Chinese Academy of Sciences (Beijing, China). N-terminal deletion mutants of this construct were created in a two-step procedure. An NheI site and a ATG start codon were inserted to Orail-
SS at sites corresponding to positions $70,74,75,76,77,79$ and 81 of hOrail by long-range PCR and followed by NheI digestion to remove NheI flanking regions. The $\Delta 273-301$ Orail-SS C-terminal deletion mutant was created by insertion of $K p n I$ sites flanking regions corresponding to positions 273 and 301 of hOrail followed by $K p n I$ digestion to remove the 273-301 corresponding region. Orail-CFP plasmid was obtained from Professor Murali Prakriya's lab at Northwestern University (Chicago, USA). The Orail-EGFP construct was created by replacing ECFP with EGFP. N-terminal deletion mutants were created using similar strategy as described for Orail-SS. NFAT-EGFP was purchased from Addgene (originally provided by Dr A. Rao) and mCherry-NFAT was created by replacing EGFP with mCherry. All point mutations were created by standard long-range PCR reaction and all plasmids were sent to the DNA Sequencing Facility at UC Berkeley for verification of the modified plasmid regions.

\section{Statistical analysis}

Statistical significance of data was calculated using one-way ANOVA with bonferroni correction (Kaleidagraph, Synergy Software) when comparing three or more data-set groups and unpaired two-tailed Student's $t$-test when comparing two data-set groups (Microsoft excel 2010).

\section{Quantitation of expression levels for Orail constructs}

For quantitation of relative expression of Orail constructs, we relied on fluorescence emitted by tagged fluorescent proteins EGFP or mCherry as a quantitative reporter of cDNA expression [60]. Images of cells expressing the indicated Orail constructs were collected with identical confocal settings. Images from untransfected cells (control) were used to asses background fluorescence values subsequently used to establish threshold. All fluorescent pixels with more than five times the threshold value were recorded and normalized to the pixel area in the analyzed field.

\section{Acknowledgments}

We would like to thank Holly Aaron and the UC Berkeley Molecular Imaging Center for technical assistance. This work was supported by an American Heart Association postdoctoral fellowship (13POST14000008 to RP) and by a grant from the National Institutes of Health (R01 NS35549 to EYI).

\section{References}

1 Peinelt C, Vig M, Koomoa DL, et al. Amplification of CRAC current by STIM1 and CRACM1 (Orai1). Nat Cell Biol 2006; 8:771-773.

2 Feske S, Gwack Y, Prakriya M, et al. A mutation in Orai1 causes immune deficiency by abrogating CRAC channel function. Nature 2006; 441:179-185.

3 Liou J, Kim ML, Heo WD, et al. STIM is a Ca2+ sensor essential for $\mathrm{Ca} 2+$-store-depletion-triggered $\mathrm{Ca} 2+$ influx. Curr Biol 2005; 15:1235-1241.

4 Roos J, DiGregorio PJ, Yeromin AV, et al. STIM1, an essential and conserved component of store-operated $\mathrm{Ca} 2+$ channel function. J Cell Biol 2005; 169:435-445.

5 Soboloff J, Spassova MA, Tang XD, Hewavitharana T, Xu W, 
Gill DL. Orail and STIM reconstitute store-operated calcium channel function. J Biol Chem 2006; 281:20661-20665.

6 Vig M, Peinelt C, Beck A, et al. CRACM1 is a plasma membrane protein essential for store-operated $\mathrm{Ca} 2+$ entry. Science 2006; 312:1220-1223.

7 Zhang SL, Yu Y, Roos J, et al. STIM1 is a Ca2+ sensor that activates CRAC channels and migrates from the $\mathrm{Ca} 2+$ store to the plasma membrane. Nature 2005; 437:902-905.

8 Zhang SL, Yeromin AV, Zhang XH-F, et al. Genome-wide RNAi screen of $\mathrm{Ca}(2+)$ influx identifies genes that regulate $\mathrm{Ca}(2+)$ release-activated $\mathrm{Ca}(2+)$ channel activity. Proc Natl Acad Sci USA 2006; 103:9357-9362.

9 Prakriya M, Feske S, Gwack Y, Srikanth S, Rao A, Hogan PG. Orail is an essential pore subunit of the CRAC channel. Nature 2006; 443:230-233.

10 Mercer JC, Dehaven WI, Smyth JT, et al. Large store-operated calcium selective currents due to co-expression of Orail or Orai2 with the intracellular calcium sensor, Stim1. J Biol Chem 2006; 281:24979-24990.

11 Williams RT, Senior PV, Van Stekelenburg L, Layton JE, Smith PJ, Dziadek MA. Stromal interaction molecule 1 (STIM1), a transmembrane protein with growth suppressor activity, contains an extracellular SAM domain modified by N-linked glycosylation. Biochim Biophys Acta 2002; 1596:131-137.

12 Stathopulos PB, Zheng L, Li GY, Plevin MJ, Ikura M. Structural and mechanistic insights into STIM1-mediated initiation of store-operated calcium entry. Cell 2008; 135:110-122.

13 Stathopulos PB, Zheng L, Ikura M. Stromal interaction molecule (STIM) 1 and STIM2 calcium sensing regions exhibit distinct unfolding and oligomerization kinetics. $J$ Biol Chem 2009; 284:728-732.

14 Park CY, Hoover PJ, Mullins FM, et al. STIM1 clusters and activates CRAC channels via direct binding of a cytosolic domain to Orai1. Cell 2009; 136:876-890.

15 Yuan JP, Zeng W, Dorwart MR, Choi YJ, Worley PF, Muallem $\mathrm{S}$. SOAR and the polybasic STIM1 domains gate and regulate Orai channels. Nat Cell Biol 2009; 11:337-343.

16 Hou X, Long SB. Functional reconstitution and structural flexibility of the CRAC channel Orai. Biophys $J$ 2015; 108:178a.

17 Hou X, Pedi L, Diver MM, Long SB. Crystal structure of the calcium release-activated calcium channel Orai. Science 2012; 338:1308-1313.

18 Thompson JL, Shuttleworth TJ. How many Orai's does it take to make a CRAC channel? Sci Rep 2013; 3:1961.

19 Baba Y, Hayashi K, Fujii Y, et al. Coupling of STIM1 to store-operated $\mathrm{Ca} 2+$ entry through its constitutive and inducible movement in the endoplasmic reticulum. Proc Natl Acad Sci USA 2006; 103:16704-16709.

20 Luik RM, Wang B, Prakriya M, Wu MM, Lewis RS. Oligomerization of STIM1 couples ER calcium depletion to CRAC channel activation. Nature 2008; 454:538-542.

21 Stathopulos PB, Li GY, Plevin MJ, Ames JB, Ikura M. Stored $\mathrm{Ca} 2+$ depletion-induced oligomerization of stromal interaction molecule 1 (STIM1) via the EF-SAM region: an initiation mechanism for capacitive Ca2+ entry. J Biol Chem 2006; 281:35855-35862.

22 Zhou Y, Srinivasan P, Razavi S, et al. Initial activation of
STIM1, the regulator of store-operated calcium entry. Nat Struct Mol Biol 2013; 20:973-981.

23 Covington ED, Wu MM, Lewis RS. Essential role for the CRAC activation domain in store-dependent oligomerization of STIM1. Mol Biol Cell 2010; 21:1897-1907.

24 Yu F, Sun L, Courjaret R, Machaca K. Role of the STIM1 C-terminal domain in STIM1 clustering. J Biol Chem 2011; 286:8375-8384.

25 Yu F, Sun L, Hubrack S, Selvaraj S, Machaca K. Intramolecular shielding maintains the ER $\mathrm{Ca}^{2+}$ sensor STIM1 in an inactive conformation. J Cell Sci 2013; 126:2401-2410.

26 Muik M, Fahrner M, Schindl R, et al. STIM1 couples to ORAI1 via an intramolecular transition into an extended conformation. EMBO J 2011; 30:1678-1689.

27 Zhou Y, Meraner P, Kwon HT, et al. STIM1 gates the store-operated calcium channel ORAI1 in vitro. Nat Struct Mol Biol 2010; 17:112-116.

28 Derler I, Plenk P, Fahrner M, et al. The extended transmembrane Orail N-terminal (ETON) region combines binding interface and gate for Orail activation by STIM1. J Biol Chem 2013; 288:29025-29034.

29 Zhou Y, Meraner P, Kwon HT, et al. STIM1 gates the store-operated calcium channel ORAI1 in vitro. Nat Struct Mol Biol 2010; 17:112-116.

30 Wu MM, Buchanan J, Luik RM, Lewis RS. Ca2+ store depletion causes STIM1 to accumulate in ER regions closely associated with the plasma membrane. J Cell Biol 2006; 174:803813.

31 McNally BA, Somasundaram A, Yamashita M, Prakriya M. Gated regulation of CRAC channel ion selectivity by STIM1. Nature 2012; 482:241-245.

32 Luik RM, Wu MM, Buchanan J, Lewis RS. The elementary unit of store-operated Ca2+ entry: local activation of CRAC channels by STIM1 at ER-plasma membrane junctions. $J$ Cell Biol 2006; 174:815-825.

33 Xu P, Lu J, Li Z, Yu X, Chen L, Xu T. Aggregation of STIM1 underneath the plasma membrane induces clustering of Orai1. Biochem Biophys Res Commun 2006; 350:969-976.

34 Lioudyno MI, Kozak JA, Penna A, et al. Orai1 and STIM1 move to the immunological synapse and are up-regulated during T cell activation. Proc Natl Acad Sci USA 2008; 105:2011-2016.

35 Sharma S, Quintana A, Findlay GM, et al. An siRNA screen for NFAT activation identifies septins as coordinators of store-operated Ca2+ entry. Nature 2013; 499:238-242.

36 McNally BA, Yamashita M, Engh A, Prakriya M. Structural determinants of ion permeation in CRAC channels. Proc Natl Acad Sci USA 2009; 106:22516-22521.

37 Takahashi Y, Murakami M, Watanabe H, et al. Essential role of the N-terminus of murine Orai1 in store-operated Ca2+ entry. Biochem Biophys Res Commun 2007; 356:45-52.

38 Hou X, Pedi L, Diver MM, Long SB. Crystal structure of the calcium release-activated calcium channel Orai. Science 2012; 338:1308-1313.

39 Lis A, Zierler S, Peinelt C, Fleig A, Penner R. A single lysine in the N-terminal region of store-operated channels is critical for STIM1-mediated gating. J Gen Physiol 2010; 136:673686.

40 Muik M, Frischauf I, Derler I, et al. Dynamic coupling of the 
putative coiled-coil domain of ORAI1 with STIM1 mediates ORAI1 channel activation. J Biol Chem 2008; 283:80148022.

41 Li Z, Lu J, Xu P, Xie X, Chen L, Xu T. Mapping the interacting domains of STIM1 and Orai1 in $\mathrm{Ca} 2+$ release-activated Ca2+ channel activation. J Biol Chem 2007; 282:2944829456.

42 Navarro-Borelly L, Somasundaram A, Yamashita M, Ren D, Miller RJ, Prakriya M. STIM1-Orai1 interactions and Orai1 conformational changes revealed by live-cell FRET microscopy. $J$ Physiol 2008; 586:5383-5401.

43 Zheng $\mathrm{H}$, Zhou M-H, Hu C, et al. Differential roles of the $\mathrm{C}$ and $\mathrm{N}$ termini of Orail protein in interacting with stromal interaction molecule 1 (STIM1) for $\mathrm{Ca} 2+$ release-activated Ca2+ (CRAC) channel activation. J Biol Chem 2013; 288:11263-11272.

44 McNally BA, Somasundaram A, Jairaman A, Yamashita M, Prakriya M. The C- and N-terminal STIM1 binding sites on Orail are required for both trapping and gating CRAC channels. J Physiol 2013; 591:2833-2850.

45 Li Z, Liu L, Deng Y, et al. Graded activation of CRAC channel by binding of different numbers of STIM1 to Orail subunits. Cell Res 2011; 21:305-315.

46 Stathopulos PB, Schindl R, Fahrner M, et al. STIM1/Orai1 coiled-coil interplay in the regulation of store-operated calcium entry. Nat Commun 2013; 4:2963.

47 Lee KP, Yuan JP, Zeng W, So I, Worley PF, Muallem S. Molecular determinants of fast $\mathrm{Ca} 2+$-dependent inactivation and gating of the Orai channels. Proc Natl Acad Sci USA 2009; 106:14687-14692.

48 Frischauf I, Muik M, Derler I, et al. Molecular determinants of the coupling between STIM1 and Orai channels: differential activation of Orai1-3 channels by a STIM1 coiled-coil mutant. J Biol Chem 2009; 284:21696-21706.

49 Calloway N, Holowka D, Baird B. A basic sequence in STIM1 promotes $\mathrm{Ca} 2+$ influx by interacting with the C-terminal acidic coiled coil of Orail. Biochemistry 2010; 49:10671071.

50 Calloway N, Vig M, Kinet JP, Holowka D, Baird B. Molecular clustering of STIM1 with Orai1/CRACM1 at the plasma membrane depends dynamically on depletion of $\mathrm{Ca} 2+$ stores and on electrostatic interactions. Mol Biol Cell 2009; 20:389399.

51 Gudlur A, Zhou Y, Hogan PG. STIM-ORAI interactions that control the CRAC channel. Curr Top Membr 2013; 71:33-58.

52 Rothberg BS, Wang Y, Gill DL. Orai channel pore properties and gating by STIM: implications from the Orai crystal structure. Sci Signal 2013; 6:pe9.

53 Sadaghiani AM, Lee SM, Odegaard JI, et al. Identification of Orail channel inhibitors by using minimal functional domains to screen small molecule microarrays. Chem Biol 2014; 21:1278-1292.

54 Gudlur A, Quintana A, Zhou Y, Hirve N, Mahapatra S, Hogan PG. STIM1 triggers a gating rearrangement at the extracellular mouth of the ORAI1 channel. Nat Commun 2014; 5:5164.

55 Zhang SL, Yeromin AV, Hu J, Amcheslavsky A, Zheng H,
Cahalan MD. Mutations in Orail transmembrane segment 1 cause STIM1-independent activation of Orail channels at glycine 98 and channel closure at arginine 91. Proc Natl Acad Sci USA 2011; 108:17838-17843.

56 Svobodobá B. CRAC channel gating-Involvement of Orai1 transmembrane regions and STIM1. Diss. Institute of Biophysics 2013.

57 Srikanth S, Yee MK, Gwack Y, Ribalet B. The third transmembrane segment of ORAI1 modulates $\mathrm{Ca} 2+$ release-activated ca2+ (CRAC) channel gating and permeation properties. J Biol Chem 2011; 286:35318-35328.

58 Lummis SCR, Beene DL, Lee LW, Lester HA, Broadhurst RW, Dougherty DA. Cis-trans isomerization at a proline opens the pore of a neurotransmitter-gated ion channel. $\mathrm{Na}$ ture 2005; 438:248-252.

59 Dang H, England PM, Farivar SS, Dougherty DA, Lester HA. Probing the role of a conserved M1 proline residue in 5-hydroxytryptamine(3) receptor gating. Mol Pharmacol 2000; 57:1114-1122.

60 Woolfson DN, Mortishire-Smith RJ, Williams DH. Conserved positioning of proline residues in membrane-spanning helices of ion-channel proteins. Biochem Biophys Res Commun 1991; 175:733-737.

61 Labro AJ, Raes AL, Bellens I, Ottschytsch N, Snyders DJ. Gating of shaker-type channels requires the flexibility of S6 caused by prolines. J Biol Chem 2003; 278:50724-50731.

62 Hackos DH, Chang T-H, Swartz KJ. Scanning the intracellular S6 activation gate in the shaker $\mathrm{K}+$ channel. J Gen Physiol 2002; 119:521-531.

63 Long SB, Tao X, Campbell EB, MacKinnon R. Atomic structure of a voltage-dependent $\mathrm{K}+$ channel in a lipid membrane-like environment. Nature 2007; 450:376-382.

64 Nesin V, Wiley G, Kousi M, et al. Activating mutations in STIM1 and ORAI1 cause overlapping syndromes of tubular myopathy and congenital miosis. Proc Natl Acad Sci USA 2014; 111:4197-4202.

65 Jha A, Ahuja M, Maléth J, et al. The STIM1 CTID domain determines access of SARAF to SOAR to regulate Orai1 channel function. $J$ Cell Biol 2013; 202:71-79.

66 Palty R, Raveh A, Kaminsky I, Meller R, Reuveny E. SARAF inactivates the store operated calcium entry machinery to prevent excess calcium refilling. Cell 2012; 149:425-438.

67 Endo Y, Noguchi S, Hara Y, et al. Dominant mutations in ORAI1 cause tubular aggregate myopathy with hypocalcemia via constitutive activation of store-operated $\mathrm{Ca} 2+$ channels. Hum Mol Genet 2015; 24:637-648.

68 Chen GJ, Qiu N, Karrer C, Caspers P, Page MG. Restriction site-free insertion of PCR products directionally into vectors. Biotechniques 2000; 28:498-500, 504-505.

69 Fersht A. Structure and Mechanism in Protein Science: a Guide to Enzyme Catalysis and Protein Folding. New York: W.H. Freemen 1999.

(Supplementary information is linked to the online version of the paper on the Cell Research website.) 